\title{
On the existence of solutions for fractional boundary value problems on the ethane graph
}

\author{
Sina Etemad ${ }^{1}$ and Shahram Rezapour ${ }^{1,2^{*}}$ (D)
}

\section{"Correspondence:}

rezapourshahram@yahoo.ca; sh.rezapour@azaruniv.ac.ir

'Department of Mathematics,

Azarbaijan Shahid Madani

University, Tabriz, Iran

${ }^{2}$ Department of Medical Research, China Medical University Hospital,

China Medical University, Taichung, Taiwan

\begin{abstract}
A few researchers have studied fractional differential equations on star graphs. They use star graphs because their method needs a common point which has edges with other nodes while other nodes have no edges between themselves. It is natural that we feel that this method is incomplete. Our aim is extending the method on more generalized graphs. In this work, we investigate the existence of solutions for some fractional boundary value problems on the ethane graph. In this way, we consider a graph with labeled vertices by 0 or 1 , inspired by a graph representation of the chemical compound of ethane, and define fractional differential equations on each edge of this graph. Also, we provide an example to illustrate our last main result.
\end{abstract}

MSC: Primary 34A08; 39A12; secondary 34B45

Keywords: Boundary value problem; Fractional differential equation; Ethane graph; Caputo derivative

\section{Introduction}

During the recent decades, initial and boundary value problems have been used in investigating natural processes in the world around us. The wide variety of such boundary value problems has attracted the attention of many researchers to study various phenomena using mathematical tools and computer simulation software. In other words, some recent publications show the importance of fractional differential equations in modeling of a variety of applied sciences (see, for example, [1-9]) and numerical computations (see, for example, [10-15]). One of our aims in this work is extending theoretical field in order to increase our abilities in finding more effective applications on chemical reactions. If we can do so, computer software engineers will be able to produce some software in the future using which everybody could do chemical experiments without the use of minerals, and this will help the environment.

The fractional calculus plays an important role in this regard. By using some techniques, we can solve the mathematical modelings described by the fractional differential equations and obtain the corresponding solutions and then analyze the qualitative behaviors of a

(c) The Author(s) 2020. This article is licensed under a Creative Commons Attribution 4.0 International License, which permits use sharing, adaptation, distribution and reproduction in any medium or format, as long as you give appropriate credit to the original author(s) and the source, provide a link to the Creative Commons licence, and indicate if changes were made. The images or other third party material in this article are included in the article's Creative Commons licence, unless indicated otherwise in a credit line to the material. If material is not included in the article's Creative Commons licence and your intended use is not permitted by statutory regulation or exceeds the permitted use, you will need to obtain permission directly from the copyright holder. To view a copy of this licence, visit http://creativecommons.org/licenses/by/4.0/. 
solution function under given boundary conditions. Existing works for such problems in general setting can be seen in the numerous published papers (see, for example, [16-51]). The graph theory is a relatively new area of mathematics in which we are concerned with networks of points connected by lines. This structure can be found in many real constructions in the world around us. In other words, by development and expansion of some dynamical and industrial systems such as gas transmission lines, water pipelines, the expansive growth of computer networks, structure of molecules in medicine and biology, etc., new descriptive models have emerged for studying the related processes designed by specialists of these fields. Due to the graph structure of these networks, the study of mathematical models described by ordinary or fractional differential equations on graphs was considered. In fact, boundary value problems on a graph are defined as a problem consisting of a system of differential equations on the given graph with certain boundary conditions on nodes.

The starting point for the theory of differential equations on graphs is related to a work of Lumer in 1980 [52]. He investigated general evolution equations on ramification spaces by using local operators defined on such spaces. With a similar structure, Nicaise studied the propagation of nerve impulses [53]. In 1989, Zavgorodnij considered boundary value problems for linear differential equations on a geometric graph where solutions of the problem were coordinated at the interior vertices [54]. He constructed an adjoint boundary value problem and obtained a self-adjointness criterion [54]. In 2008, Gordeziani et al. discussed the existence and uniqueness of solutions for ordinary differential equations on graphs [55]. They used the double-sweep method for solving the boundary value problem and presented a numerical approach.

In most of the mentioned works, differential equations are considered on a graph, and solutions of them are obtained by computational and numerical approaches. But there are limited works in fractional boundary value problems on graphs in which the existence of solutions is proved by some techniques in fixed point theory [56, 57]. The first work in this regard is related to a paper of Graef et al. [56]. The authors introduced a star graph $G=$ $V(G) \cup E(G)$ consisting of three vertices and two edges with $V(G)=\left\{v_{0}, v_{1}, v_{2}\right\}$ and $E(G)=$ $\left\{e_{1}=\overrightarrow{v_{1} v_{0}}, e_{2}=\overrightarrow{v_{2} v_{0}}\right\}$, where $v_{0}$ is the junction node and $e_{i}=\overrightarrow{v_{i} v_{0}}$ is the edge connecting nodes $v_{i}$ to $v_{0}$ with length $l_{i}=\left|\overrightarrow{v_{i} v_{0}}\right|$ for $i=1,2$. On each edge $e_{i}=\overrightarrow{v_{i} v_{0}}$, a local coordinate system with origin at vertices $v_{1}$ and $v_{2}$ and the coordinate $t \in\left(0, l_{i}\right)$ is considered. Graef et al. defined a system of nonlinear fractional differential equations on each $e_{i}=\overrightarrow{v_{i} v_{0}}$ by

$$
-\mathcal{D}_{0}^{\alpha} u_{i}(t)=g_{i}(t) h_{i}\left(t, u_{i}(t)\right) \quad\left(i=1,2, t \in\left(0, l_{i}\right)\right)
$$

with boundary value conditions

$$
u_{1}(0)=u_{2}(0)=0, \quad u_{1}\left(l_{1}\right)=u_{2}\left(l_{2}\right), \quad \mathcal{D}_{0}^{\beta} u_{1}\left(l_{1}\right)+\mathcal{D}_{0}^{\beta} u_{2}\left(l_{2}\right)=0,
$$

where $\alpha \in(1,2], \beta \in(0, \alpha), g_{i}:\left[0, l_{i}\right] \rightarrow \mathbb{R}$ are continuous functions with $g_{i}(t) \neq 0$ on $\left[0, l_{i}\right]$ and also $h_{i}:\left[0, l_{i}\right] \times \mathbb{R} \rightarrow \mathbb{R}$ are continuous functions. Two fractional operators $\mathcal{D}_{0}^{\alpha}$ and $\mathcal{D}_{0}^{\beta}$ denote Riemann-Liouville fractional derivatives. The Banach contraction principle and the Schauder fixed point theorem are applied to establish the existence results by authors.

In 2019, Mehandiratta et al. generalized the work of Graef to the case of a star graph consisting of $n+1$ vertices and $n$ edges [57]. Indeed, the authors considered a general star 
graph $G$ with $V(G)=\left\{v_{0}, v_{1}, \ldots, v_{n}\right\}$ and $E(G)=\left\{e_{1}=\overrightarrow{v_{1} v_{0}}, e_{2}=\overrightarrow{v_{2} v_{0}}, \ldots, e_{n}=\overrightarrow{v_{n} v_{0}}\right\}$, where $l_{i}=\left|\overrightarrow{v_{i} v_{0}}\right|$ is the length of each edge $e_{i}$ connecting vertices $v_{i}$ to $v_{0}(i=1,2, \ldots, n)$. The authors investigated the following boundary value problems on each edge of the star graph $G$ given by

$$
\left\{\begin{array}{l}
{ }^{c} \mathcal{D}_{0}^{\alpha} u_{i}(t)=f_{i}\left(t, u_{i}(t),{ }^{c} \mathcal{D}_{0}^{\beta} u_{i}(t)\right) \quad\left(i=1,2, \ldots, n, t \in\left(0, l_{i}\right)\right) \\
u_{i}(0)=0, \quad u_{i}\left(l_{i}\right)=u_{j}\left(l_{j}\right) \quad(i \neq j) \\
\sum_{i=1}^{n} u_{i}^{\prime}\left(l_{i}\right)=0
\end{array}\right.
$$

where $\alpha \in(1,2], \beta \in(0, \alpha-1], f_{i}:\left[0, l_{i}\right] \times \mathbb{R} \times \mathbb{R} \rightarrow \mathbb{R}$ are continuous functions and ${ }^{c} \mathcal{D}_{0}^{\gamma}$ denotes the Caputo fractional derivative of order $\gamma \in\{\alpha, \beta\}$. They used transformations $x=\frac{t}{l_{i}} \in[0,1]$ and $z(x)=u(t)=u\left(l_{i} x\right)$ for $t \in\left[0, l_{i}\right]$ and proved the relation ${ }^{c} \mathcal{D}_{0}^{\alpha} u(t)=$ $l_{i}^{-\alpha}\left({ }^{c} \mathcal{D}_{0}^{\alpha} z(x)\right)$. Then, by applying these transformations, the system of fractional boundary value problems on graph (1) converts into the following system of fractional boundary value problems on the unit interval $[0,1]$ given by

$$
{ }^{c} \mathcal{D}_{0}^{\alpha} z_{i}(x)=l_{i}^{\alpha} h_{i}\left(x, z_{i}(x), l_{i}^{-\beta}{ }^{\beta} \mathcal{D}_{0}^{\beta} z_{i}(x)\right) \quad(x \in[0,1])
$$

with boundary conditions $z_{i}(0)=0, z_{i}(1)=z_{j}(1)$ for $i \neq j$ and $\sum_{i=1}^{n} l_{i}^{-1} z_{i}^{\prime}(1)=0$, where $z_{i}(x)=$ $u_{i}\left(l_{i} x\right)$ and $h_{i}(x, u, w)=f_{i}\left(l_{i} x, u, w\right)$ for $i=1,2, \ldots, n$.

Motivated by the aforementioned works, our aim is to generalize the above boundary value problems to a new problem on the ethane graph which is a general graph with respect to star graphs. More precisely, by considering the ethane graph with labeled vertices by 0 or 1 (see Fig. 6), we investigate the existence of solutions for the nonlinear fractional boundary value problem

$$
\left\{\begin{array}{l}
{ }^{c} \mathcal{D}_{0}^{\vartheta} u_{i}(t)=h_{i}\left(t, u_{i}(t), u_{i}^{\prime}(t)\right) \quad(t \in[0,1], i=1,2, \ldots, 7) \\
\lambda_{1} u_{i}(0)+\lambda_{2} u_{i}(1)=\lambda_{3} \int_{0}^{1} u_{i}(s) \mathrm{d} s, \quad \lambda_{1} u_{i}^{\prime}(0)+\lambda_{2} u_{i}^{\prime}(1)=\lambda_{3} \int_{0}^{1} u_{i}(s) \mathrm{d} s
\end{array}\right.
$$

where $\vartheta \in(1,2], \lambda_{1}, \lambda_{2}, \lambda_{3} \in \mathbb{R},{ }^{c} \mathcal{D}_{0}^{\vartheta}$ denotes the Caputo fractional derivative and functions $h_{i}:[0,1] \times \mathbb{R} \times \mathbb{R} \rightarrow \mathbb{R}$ are continuous for $i=1,2, \ldots, 7$, where $n=7$ is the number of edges of the graph representation of ethane with $\left|e_{i}\right|=1$.

The boundary conditions in this problem show that the linear combinations of values of the unknown functions and their derivatives at two ends of each edge are proportional to a multiple of the integral of the unknown functions. Also, it is notable that the obtained solutions for the fractional boundary value problem (2) can be interpreted in different practical meanings of the organic chemistry. In other words, every solution function $u_{i}(t)$ on an arbitrary edge $e_{i}$ may indicate an amount of the bond energy, the bond strength, the bond polarity, etc. This could lead to valuable applications in chemical reactions theory. Hence, we claim that this abstract idea could be useful for young researchers in their future work.

This paper is arranged as follows. In Sect. 2, some primitive notions for labeling the ethane graph by 0 or 1 are explained. Moreover, some necessary relations on the fractional calculus are recalled. In Sect. 3, the main existence results for the nonlinear fractional boundary value problem (2) are proved by known fixed point theorems. In the end of the paper, an example is provided to illustrate the validity of results. 
Figure 1 A sketch of the star graph $G$ with two edges

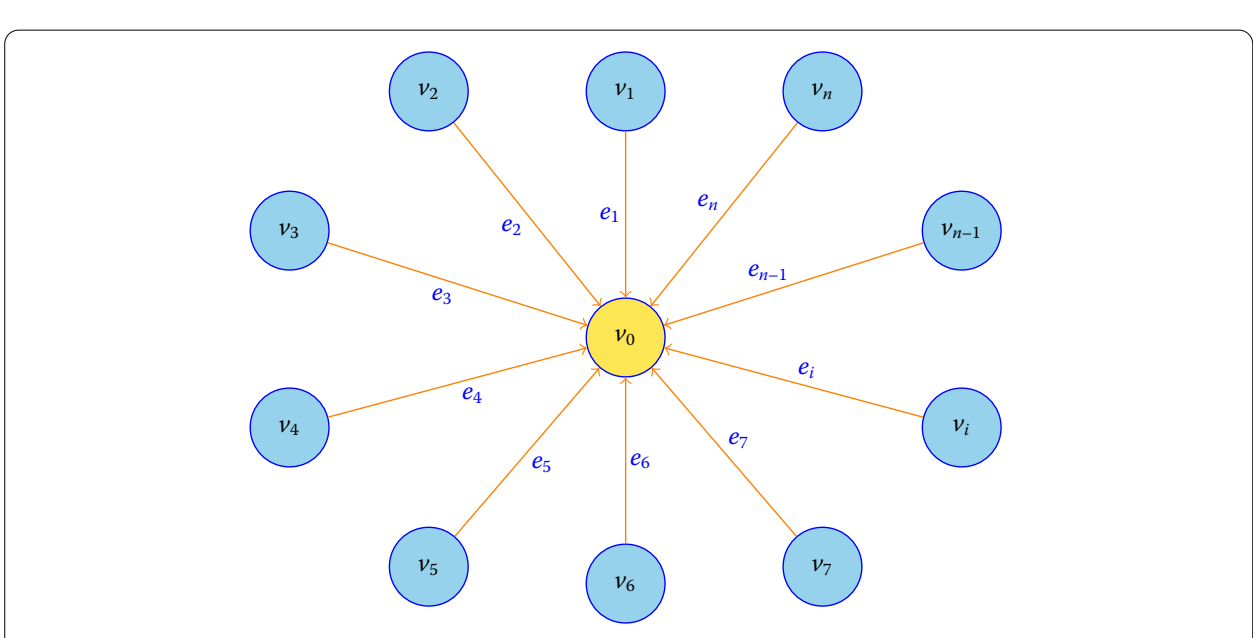

Figure 2 A sketch of the star graph $G$ with $n$ edges

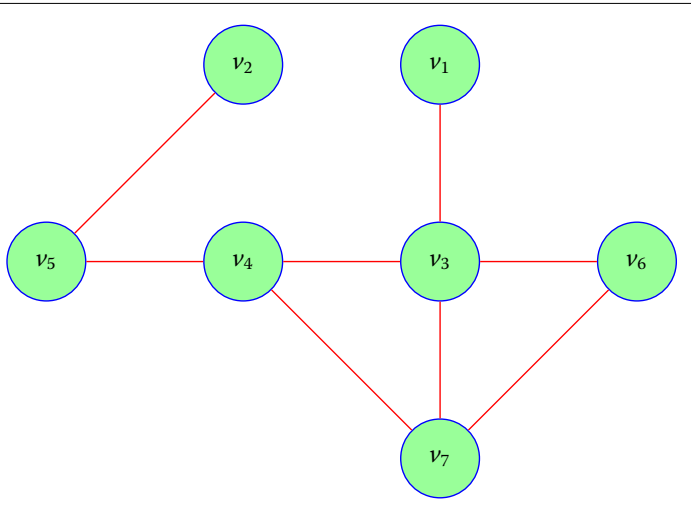

Figure 3 A sketch of the non-star graph $G$ with more than one junction node

\section{Preliminaries}

In this section, we provide some primitive concepts about the new class of fractional boundary value problems (2) on the ethane graph. For this, we first state two important points about the used methods in [56] and [57].

(1) In both articles, the authors assume the graph $G$ as a star graph consisting of one junction node $v_{0}$ (Figs. 1 and 2), while in general cases, the graph $G$ may not be in the form of a star graph and may have a general structure with more than one junction node. For example, see Fig. 3 where there exist five junction nodes.

(2) In both articles, the authors consider the length of each edge to be the variable values $\left|e_{i}\right|=l_{i}$ for $i=1,2, \ldots, n$, where $n$ denotes the number of edges for the graph $G$. Next, they convert $\left[0, l_{i}\right]$ into a unit interval $[0,1]$ by using a change of variable for the normalization 


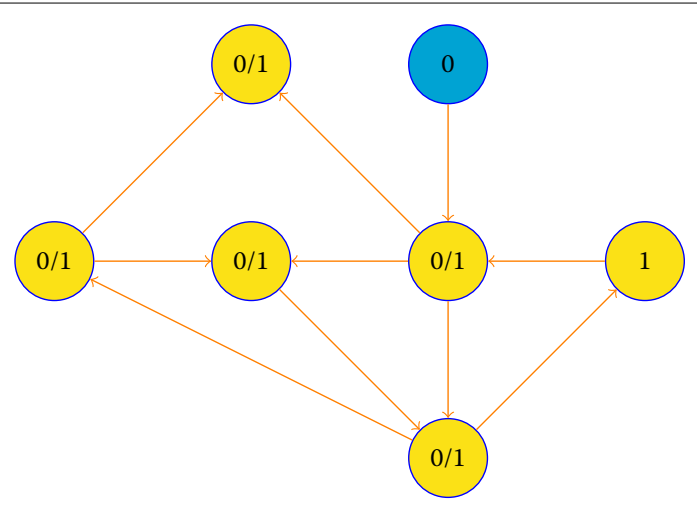

Figure 4 A sketch of the general graph $G$ with labeled vertices

Figure 5 A sketch of the graph representation of ethane $\left(\mathrm{C}_{2} \mathrm{H}_{6}\right)$

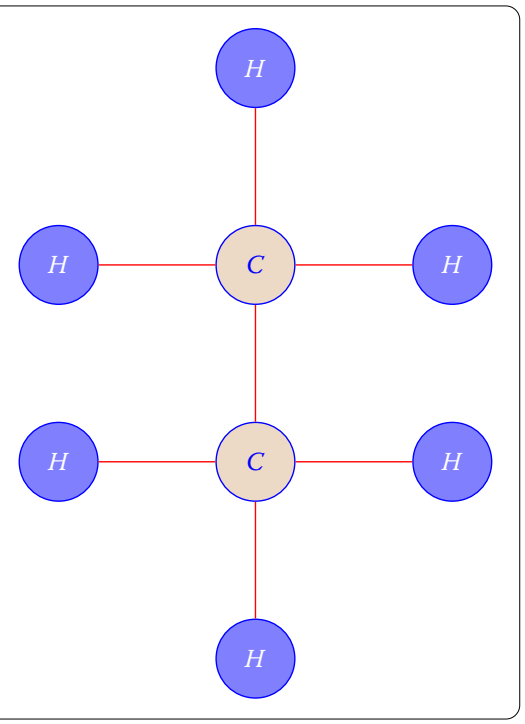

of the length of all edges, while from the beginning, one can consider the length of all edges to be fixed value $\left|e_{i}\right|=1$ without specifying boundary vertices of each edge as the origin. For this purpose, we propose a new method for labeling vertices. In this case, we can assign two labels 0 or 1 to each vertex of a graph. In other words, the label of each vertex depends on the direction of the corresponding edge. When we move along an arbitrary edge, the label of the starting vertex and the ending vertex are considered values 0 and 1 and vice versa. Hence, some vertices may have two labels 0 and 1 simultaneously, and the origin of each edge is not fixed, and it changes whenever the direction of the movement along the edge is changed. By this rule, we do not need to normalize the length of each edge by using the specific transformation, and also we are free to determine one of two vertices of the corresponding edge as the origin. For example, see Fig. 4 illustrating one of the possible cases for labeling. In this graph, we begin to move along edges from the blue vertex in the first step.

In this paper, we are going to study some existence results for a system of fractional differential equations on the ethane graph. We represent the ethane molecule as a graph with labeled vertices by 0 or 1 . As you know, ethane is a chemical compound of hydrogen $(\mathrm{H})$ and carbon $(\mathrm{C})$ with chemical formula $\mathrm{C}_{2} \mathrm{H}_{6}$ (see Fig. 5). Ethane is the simplest hy- 
Figure 6 A sketch of the graph representation of ethane with labeled vertices by 0 or 1

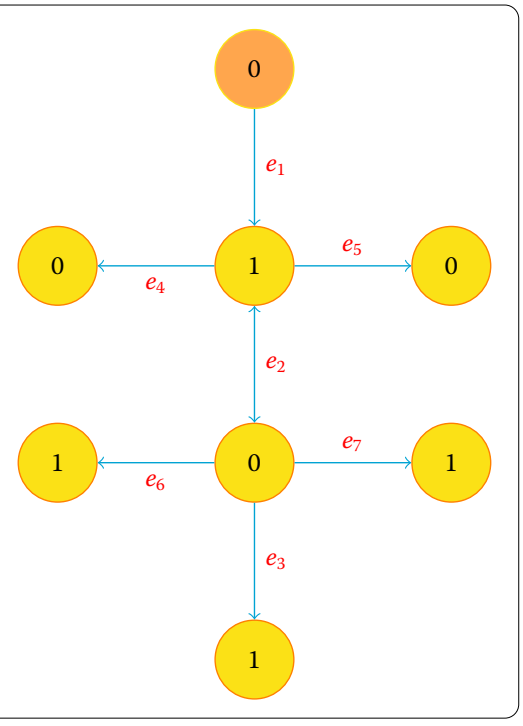

drocarbon that contains a single carbon-carbon bond. We consider atoms of hydrogens and carbons as vertices of the graph and also the existing chemical bonds between atoms are considered as edges of the graph. This molecular graph is not a star graph, and so the method proposed in articles [56] and [57] for assigning the origin at boundary nodes except the junction node $v_{0}$ will not be useful because this graph has more than one junction node. Thus we have to use a different method. We can label vertices of the above graph in the form of labeled vertices by two values 0 or 1 and consider the length of each edge to be unit value $l_{i}=1$ (see Fig. 6). For more complicate graphs, one can use doubled indexing for nodes of graphs.

Hence, in view of the ethane graph with labeled vertices by 0 or 1 as above, we can perform our purposes on the existence of solutions for the nonlinear fractional boundary value problem (2).

Now, we recall some basic notions and properties about the fractional calculus. Let $\vartheta>0$. The Riemann-Liouville fractional integral of a function $u:[a, b] \rightarrow \mathbb{R}$ is defined by

$$
\mathcal{I}_{0}^{\vartheta} u(t)=\int_{0}^{t} \frac{(t-s)^{\vartheta-1}}{\Gamma(\vartheta)} u(s) \mathrm{d} s
$$

provided that the right-hand side integral exists $[58,59]$. Let $n-1 \leq \vartheta<n$. Then $n=[\vartheta]+1$. The Caputo fractional derivative of a function $u \in C^{(n)}([a, b], \mathbb{R})$ is defined by ${ }^{c} \mathcal{D}_{0}^{\vartheta} u(t)=$ $\int_{0}^{t} \frac{(t-s)^{n-\vartheta-1}}{\Gamma(n-\vartheta)} u^{(n)}(s) \mathrm{d} s$ provided that the integral exists $[58,59]$. It has been proved that the general solution for the homogeneous fractional differential equation ${ }^{c} \mathcal{D}_{0}^{\vartheta} u(t)=0$ is in the form $u(t)=b_{0}^{*}+b_{1}^{*} t+b_{2}^{*} t^{2}+\cdots+b_{n-1}^{*} t^{n-1}$, and we have

$$
\mathcal{I}_{0}^{\vartheta}\left({ }^{c} \mathcal{D}_{0}^{\vartheta} u(t)\right)=u(t)+\sum_{j=0}^{n-1} b_{j}^{*} t^{j}=u(t)+b_{0}^{*}+b_{1}^{*} t+b_{2}^{*} t^{2}+\cdots+b_{n-1}^{*} t^{n-1},
$$

where $b_{0}^{*}, \ldots, b_{n-1}^{*}$ are some real constants [60]. We need the next results which are known as Schaefer and Krasnoselskii fixed point theorems, respectively. 
Lemma 1 ([61]) Let $\mathcal{X}$ be a Banach space and $\Upsilon: \mathcal{X} \rightarrow \mathcal{X}$ be a completely continuous operator. Then either the set $\{u \in \mathcal{X}: u=\mu \Upsilon u, \mu \in(0,1)\}$ is unbounded or the operator $\Upsilon$ has at least one fixed point in $\mathcal{X}$.

Lemma 2 ([61]) Let $\mathcal{E}$ be a closed, bounded, convex, and nonempty subset of a Banach space $\mathcal{X}$. Let $\Upsilon_{1}$ and $\Upsilon_{2}$ be two operators such that $\Upsilon_{1} u+\Upsilon_{2} v \in \mathcal{E}$ whenever $u, v \in \mathcal{E}, \Upsilon_{1}$ is compact and continuous and $\Upsilon_{2}$ is a contraction map. Then there exists $w \in \mathcal{E}$ such that $w=\Upsilon_{1} w+\Upsilon_{2} w$.

\section{Main results}

In this section, we prove our main results on the ethane graph (Fig. 6). In this way, we consider the Banach spaces $\mathcal{X}_{i}=\left\{u_{i}: u_{i}, u_{i}^{\prime} \in C[0,1]\right\}$ with the norm $\left\|u_{i}\right\|_{\mathcal{X}_{i}}=\sup _{t \in[0,1]}\left|u_{i}(t)\right|+$ $\sup _{t \in[0,1]}\left|u_{i}^{\prime}(t)\right|$ for $i=1,2, \ldots, 7$. Note that the product space $\mathcal{X}=\left(\mathcal{X}_{1}, \mathcal{X}_{2}, \ldots, \mathcal{X}_{7}\right)$ equipped with the norm $\left\|u=\left(u_{1}, u_{2}, \ldots, u_{7}\right)\right\|_{\mathcal{X}}=\sum_{i=1}^{7}\left\|u_{i}\right\|_{\mathcal{X}_{i}}$ is a Banach space.

Lemma 3 Let $\varphi_{1}, \ldots, \varphi_{7} \in C[0,1]$. Then $u_{i}^{*}$ is a solution for the boundary value problem

$$
\left\{\begin{array}{l}
{ }^{c} \mathcal{D}_{0}^{\vartheta} u_{i}(t)=\varphi_{i}(t) \quad(t \in[0,1]), \\
\lambda_{1} u_{i}(0)+\lambda_{2} u_{i}(1)=\lambda_{3} \int_{0}^{1} u_{i}(s) \mathrm{d} s, \quad \lambda_{1} u_{i}^{\prime}(0)+\lambda_{2} u_{i}^{\prime}(1)=\lambda_{3} \int_{0}^{1} u_{i}(s) \mathrm{d} s,
\end{array}\right.
$$

if and only if $u_{i}^{*}$ is a solution for the following fractional integral equation satisfying the boundary conditions:

$$
\begin{aligned}
u_{i}(t)= & \int_{0}^{t} \frac{(t-s)^{\vartheta-1}}{\Gamma(\vartheta)} \varphi_{i}(s) \mathrm{d} s \\
& +\frac{\Lambda_{1}-\lambda_{2} \lambda_{3}\left(\lambda_{1}+\lambda_{2}-\lambda_{3}\right) t}{\left(\lambda_{1}+\lambda_{2}-\lambda_{3}\right) \Lambda} \int_{0}^{1} \frac{(1-s)^{\vartheta-1}}{\Gamma(\vartheta)} \varphi_{i}(s) \mathrm{d} s \\
& +\frac{\Lambda_{2}-\lambda_{2}\left(\lambda_{1}+\lambda_{2}-\lambda_{3}\right)^{2} t}{\left(\lambda_{1}+\lambda_{2}-\lambda_{3}\right) \Lambda} \int_{0}^{1} \frac{(1-s)^{\vartheta-2}}{\Gamma(\vartheta-1)} \varphi_{i}(s) \mathrm{d} s \\
& +\frac{\Lambda_{3}+\left(\lambda_{1}+\lambda_{2}\right) \lambda_{3}\left(\lambda_{1}+\lambda_{2}-\lambda_{3}\right) t}{\left(\lambda_{1}+\lambda_{2}-\lambda_{3}\right) \Lambda} \int_{0}^{1} \int_{0}^{s} \frac{(s-\tau)^{\vartheta-1}}{\Gamma(\vartheta)} \varphi_{i}(\tau) \mathrm{d} \tau \mathrm{d} s,
\end{aligned}
$$

where

$$
\begin{aligned}
& \Lambda=\lambda_{1}^{2}+\lambda_{2}^{2}+2 \lambda_{1} \lambda_{2}-\frac{3}{2} \lambda_{1} \lambda_{3}-\frac{1}{2} \lambda_{2} \lambda_{3} \neq 0, \\
& \Lambda_{1}=\frac{3}{2} \lambda_{2}^{2} \lambda_{3}-\frac{1}{2} \lambda_{2} \lambda_{3}^{2}-\lambda_{1}^{2} \lambda_{2}-\lambda_{2}^{3}-2 \lambda_{1} \lambda_{2}^{2}+\frac{3}{2} \lambda_{1} \lambda_{2} \lambda_{3}, \\
& \Lambda_{2}=\lambda_{1} \lambda_{2}^{2}+\lambda_{2}^{3}-\frac{3}{2} \lambda_{2}^{2} \lambda_{3}-\frac{1}{2} \lambda_{1} \lambda_{2} \lambda_{3}+\frac{1}{2} \lambda_{2} \lambda_{3}^{2}, \\
& \Lambda_{3}=\lambda_{1}^{2} \lambda_{3}+\lambda_{1} \lambda_{2} \lambda_{3}-\lambda_{1} \lambda_{3}^{2} .
\end{aligned}
$$

Proof Let $u_{i}^{*}$ be a solution for the fractional problem (3) $(i=1,2, \ldots, 7)$. Then there exist constants $b_{0}^{(i)}, b_{1}^{(i)} \in \mathbb{R}$ such that $u_{i}^{*}(t)=\mathcal{I}_{0}^{\vartheta} \varphi_{i}(t)+b_{0}^{(i)}+b_{1}^{(i)} t$. In other words,

$$
u_{i}^{*}(t)=\int_{0}^{t} \frac{(t-s)^{\vartheta-1}}{\Gamma(\vartheta)} \varphi_{i}(s) \mathrm{d} s+b_{0}^{(i)}+b_{1}^{(i)} t .
$$


Thus, $u_{i}^{*^{\prime}}(t)=\int_{0}^{t} \frac{(t-s)^{\vartheta-2}}{\Gamma(\vartheta-1)} \varphi_{i}(s) \mathrm{d} s+b_{1}^{(i)}$ and so

$$
\int_{0}^{1} u_{i}^{*}(s) \mathrm{d} s=\int_{0}^{1} \int_{0}^{s} \frac{(s-\tau)^{\vartheta-1}}{\Gamma(\vartheta)} \varphi_{i}(\tau) \mathrm{d} \tau \mathrm{d} s+b_{0}^{(i)}+\frac{1}{2} b_{1}^{(i)} .
$$

By using the boundary conditions, we obtain

$$
\begin{aligned}
b_{0}^{(i)}= & \frac{\Lambda_{1}}{\left(\lambda_{1}+\lambda_{2}-\lambda_{3}\right) \Lambda} \int_{0}^{1} \frac{(1-s)^{\vartheta-1}}{\Gamma(\vartheta)} \varphi_{i}(s) \mathrm{d} s \\
& +\frac{\Lambda_{2}}{\left(\lambda_{1}+\lambda_{2}-\lambda_{3}\right) \Lambda} \int_{0}^{1} \frac{(1-s)^{\vartheta-2}}{\Gamma(\vartheta-1)} \varphi_{i}(s) \mathrm{d} s \\
& +\frac{\Lambda_{3}}{\left(\lambda_{1}+\lambda_{2}-\lambda_{3}\right) \Lambda} \int_{0}^{1} \int_{0}^{s} \frac{(s-\tau)^{\vartheta-1}}{\Gamma(\vartheta)} \varphi_{i}(\tau) \mathrm{d} \tau \mathrm{d} s
\end{aligned}
$$

and

$$
\begin{aligned}
b_{1}^{(i)}= & -\frac{\lambda_{2} \lambda_{3}}{\Lambda} \int_{0}^{1} \frac{(1-s)^{\vartheta-1}}{\Gamma(\vartheta)} \varphi_{i}(s) \mathrm{d} s-\frac{\lambda_{2}\left(\lambda_{1}+\lambda_{2}-\lambda_{3}\right)}{\Lambda} \\
& \times \int_{0}^{1} \frac{(1-s)^{\vartheta-2}}{\Gamma(\vartheta-1)} \varphi_{i}(s) \mathrm{d} s+\frac{\left(\lambda_{1}+\lambda_{2}\right) \lambda_{3}}{\Lambda} \int_{0}^{1} \int_{0}^{s} \frac{(s-\tau)^{\vartheta-1}}{\Gamma(\vartheta)} \varphi_{i}(\tau) \mathrm{d} \tau \mathrm{d} s .
\end{aligned}
$$

Now, by substituting the values for $b_{0}^{(i)}$ and $b_{1}^{(i)}$ in equation (5), one can find that $u_{i}^{*}$ is a solution for integral equation (4). For the converse part, by using some direct calculations, one can see that $u_{i}^{*}$ is a solution for the fractional problem (3) whenever $u_{i}^{*}$ is a solution for integral equation (5). This completes the proof.

Now, by considering Lemma 3, define the operator $\Upsilon: \mathcal{X} \rightarrow \mathcal{X}$ by

$$
\Upsilon\left(u_{1}, u_{2}, \ldots, u_{7}\right)(t):=\left(\Upsilon_{1}\left(u_{1}, u_{2}, \ldots, u_{7}\right)(t), \ldots, \Upsilon_{7}\left(u_{1}, u_{2}, \ldots, u_{7}\right)(t)\right)
$$

where

$$
\begin{aligned}
\Upsilon_{i}\left(u_{1}, u_{2}, \ldots, u_{7}\right)(t)= & \int_{0}^{t} \frac{(t-s)^{\vartheta-1}}{\Gamma(\vartheta)} h_{i}\left(s, u_{i}(s), u_{i}^{\prime}(s)\right) \mathrm{d} s \\
& +\frac{\Lambda_{1}-\lambda_{2} \lambda_{3}\left(\lambda_{1}+\lambda_{2}-\lambda_{3}\right) t}{\left(\lambda_{1}+\lambda_{2}-\lambda_{3}\right) \Lambda} \int_{0}^{1} \frac{(1-s)^{\vartheta-1}}{\Gamma(\vartheta)} h_{i}\left(s, u_{i}(s), u_{i}^{\prime}(s)\right) \mathrm{d} s \\
& +\frac{\Lambda_{2}-\lambda_{2}\left(\lambda_{1}+\lambda_{2}-\lambda_{3}\right)^{2} t}{\left(\lambda_{1}+\lambda_{2}-\lambda_{3}\right) \Lambda} \int_{0}^{1} \frac{(1-s)^{\vartheta-2}}{\Gamma(\vartheta-1)} h_{i}\left(s, u_{i}(s), u_{i}^{\prime}(s)\right) \mathrm{d} s \\
& +\frac{\Lambda_{3}+\left(\lambda_{1}+\lambda_{2}\right) \lambda_{3}\left(\lambda_{1}+\lambda_{2}-\lambda_{3}\right) t}{\left(\lambda_{1}+\lambda_{2}-\lambda_{3}\right) \Lambda} \\
& \times \int_{0}^{1} \int_{0}^{s} \frac{(s-\tau)^{\vartheta-1}}{\Gamma(\vartheta)} h_{i}\left(\tau, u_{i}(\tau), u_{i}^{\prime}(\tau)\right) \mathrm{d} \tau \mathrm{d} s
\end{aligned}
$$


for all $t \in[0,1]$ and $u_{i} \in \mathcal{X}_{i}$. Put

$$
\begin{aligned}
\mathcal{K}_{0}^{*}:= & \frac{1}{\Gamma(\vartheta+1)}+\frac{\left|\Lambda_{1}\right|+\left|\lambda_{2}\right|\left|\lambda_{3}\right|\left|\lambda_{1}+\lambda_{2}-\lambda_{3}\right|}{\left|\lambda_{1}+\lambda_{2}-\lambda_{3}\right||\Lambda| \Gamma(\vartheta+1)} \\
& +\frac{\left|\Lambda_{2}\right|+\left|\lambda_{2}\right|\left(\lambda_{1}+\lambda_{2}-\lambda_{3}\right)^{2}}{\left|\lambda_{1}+\lambda_{2}-\lambda_{3}\right||\Lambda| \Gamma(\vartheta)} \\
& +\frac{\left|\Lambda_{3}\right|+\left|\lambda_{1}+\lambda_{2}\right|\left|\lambda_{3}\right|\left|\lambda_{1}+\lambda_{2}-\lambda_{3}\right|}{\left|\lambda_{1}+\lambda_{2}-\lambda_{3}\right||\Lambda| \Gamma(\vartheta+2)}, \\
\mathcal{K}_{1}^{*}:= & \frac{1}{\Gamma(\vartheta)}+\frac{\left|\lambda_{2}\right|\left|\lambda_{3}\right|\left|\lambda_{1}+\lambda_{2}-\lambda_{3}\right|}{\left|\lambda_{1}+\lambda_{2}-\lambda_{3}\right||\Lambda| \Gamma(\vartheta+1)}+\frac{\left|\lambda_{2}\right|\left(\lambda_{1}+\lambda_{2}-\lambda_{3}\right)^{2}}{\left|\lambda_{1}+\lambda_{2}-\lambda_{3}\right||\Lambda| \Gamma(\vartheta)} \\
& +\frac{\left|\lambda_{1}+\lambda_{2}\right|\left|\lambda_{3}\right|\left|\lambda_{1}+\lambda_{2}-\lambda_{3}\right|}{\left|\lambda_{1}+\lambda_{2}-\lambda_{3}\right||\Lambda| \Gamma(\vartheta+2)}, \\
\Delta_{1}^{*}:= & \frac{\left|\Lambda_{1}\right|+\left|\lambda_{2}\right|\left|\lambda_{3}\right|\left|\lambda_{1}+\lambda_{2}-\lambda_{3}\right|}{\left|\lambda_{1}+\lambda_{2}-\lambda_{3}\right||\Lambda| \Gamma(\vartheta+1)}+\frac{\left|\Lambda_{2}\right|+\left|\lambda_{2}\right|\left(\lambda_{1}+\lambda_{2}-\lambda_{3}\right)^{2}}{\left|\lambda_{1}+\lambda_{2}-\lambda_{3}\right||\Lambda| \Gamma(\vartheta)} \\
& +\frac{\left|\Lambda_{3}\right|+\left|\lambda_{1}+\lambda_{2}\right|\left|\lambda_{3}\right|\left|\lambda_{1}+\lambda_{2}-\lambda_{3}\right|}{\left|\lambda_{1}+\lambda_{2}-\lambda_{3}\right||\Lambda| \Gamma(\vartheta+2)}, \\
\Delta_{2}^{*}:= & \frac{\left|\lambda_{2}\right|\left|\lambda_{3}\right|\left|\lambda_{1}+\lambda_{2}-\lambda_{3}\right|}{\left|\lambda_{1}+\lambda_{2}-\lambda_{3}\right||\Lambda| \Gamma(\vartheta+1)}+\frac{\left|\lambda_{2}\right|\left(\lambda_{1}+\lambda_{2}-\lambda_{3}\right)^{2}}{\left|\lambda_{1}+\lambda_{2}-\lambda_{3}\right||\Lambda| \Gamma(\vartheta)} \\
& +\frac{\left|\lambda_{1}+\lambda_{2}\right|\left|\lambda_{3}\right|\left|\lambda_{1}+\lambda_{2}-\lambda_{3}\right|}{\left|\lambda_{1}+\lambda_{2}-\lambda_{3}\right||\Lambda| \Gamma(\vartheta+2)} .
\end{aligned}
$$

Theorem 4 Let $h_{1}, \ldots, h_{7}:[0,1] \times \mathbb{R} \times \mathbb{R} \rightarrow \mathbb{R}$ be continuous functions. Assume that there exist constants $L_{i}>0$ such that $\left|h_{i}\left(t, u_{1}, u_{2}\right)\right| \leq L_{i}$ for all $u_{1}, u_{2} \in \mathbb{R}$ and $t \in[0,1]$ $(i=1,2, \ldots, 7)$. Then the fractional boundary value problem (2) has a solution.

Proof By considering definition of the operator $\Upsilon$, it is clear that the fractional boundary value problem (2) has a solution if and only if $\Upsilon$ has a fixed point on the product space $\mathcal{X}=\mathcal{X}_{1} \times \cdots \times \mathcal{X}_{7}$. First, we show that the operator $\Upsilon$ is complete continuous. Since the functions $h_{1}, \ldots, h_{7}$ are continuous, the operator $\Upsilon: \mathcal{X} \rightarrow \mathcal{X}$ is continuous. Let $M$ be a bounded subset of $\mathcal{X}$ and $u=\left(u_{1}, u_{2}, \ldots, u_{7}\right) \in \mathcal{X}$. Then we have

$$
\begin{aligned}
\left|\Upsilon_{i} u(t)\right| \leq & \int_{0}^{t} \frac{(t-s)^{\vartheta-1}}{\Gamma(\vartheta)}\left|h_{i}\left(s, u_{i}(s), u_{i}^{\prime}(s)\right)\right| \mathrm{d} s \\
& +\frac{\left|\Lambda_{1}\right|+\left|\lambda_{2}\right|\left|\lambda_{3}\right|\left|\lambda_{1}+\lambda_{2}-\lambda_{3}\right| t}{\left|\lambda_{1}+\lambda_{2}-\lambda_{3}\right||\Lambda|} \int_{0}^{1} \frac{(1-s)^{\vartheta-1}}{\Gamma(\vartheta)}\left|h_{i}\left(s, u_{i}(s), u_{i}^{\prime}(s)\right)\right| \mathrm{d} s \\
& +\frac{\left|\Lambda_{2}\right|+\left|\lambda_{2}\right|\left(\lambda_{1}+\lambda_{2}-\lambda_{3}\right)^{2} t}{\left|\lambda_{1}+\lambda_{2}-\lambda_{3}\right||\Lambda|} \int_{0}^{1} \frac{(1-s)^{\vartheta-2}}{\Gamma(\vartheta-1)}\left|h_{i}\left(s, u_{i}(s), u_{i}^{\prime}(s)\right)\right| \mathrm{d} s \\
& +\frac{\left|\Lambda_{3}\right|+\left|\lambda_{1}+\lambda_{2}\right|\left|\lambda_{3}\right|\left|\lambda_{1}+\lambda_{2}-\lambda_{3}\right| t}{\left|\lambda_{1}+\lambda_{2}-\lambda_{3}\right||\Lambda|} \\
& \times \int_{0}^{1} \int_{0}^{s} \frac{(s-\tau)^{\vartheta-1}}{\Gamma(\vartheta)}\left|h_{i}\left(\tau, u_{i}(\tau), u_{i}^{\prime}(\tau)\right)\right| \mathrm{d} \tau \mathrm{d} s \\
\leq & \int_{0}^{t} \frac{(t-s)^{\vartheta-1}}{\Gamma(\vartheta)} L_{i} \mathrm{~d} s+\frac{\left|\Lambda_{1}\right|+\left|\lambda_{2}\right|\left|\lambda_{3}\right|\left|\lambda_{1}+\lambda_{2}-\lambda_{3}\right| t}{\left|\lambda_{1}+\lambda_{2}-\lambda_{3}\right||\Lambda|} \int_{0}^{1} \frac{(1-s)^{\vartheta-1}}{\Gamma(\vartheta)} L_{i} \mathrm{~d} s \\
& +\frac{\left|\Lambda_{2}\right|+\left|\lambda_{2}\right|\left(\lambda_{1}+\lambda_{2}-\lambda_{3}\right)^{2} t}{\left|\lambda_{1}+\lambda_{2}-\lambda_{3}\right||\Lambda|} \int_{0}^{1} \frac{(1-s)^{\vartheta-2}}{\Gamma(\vartheta-1)} L_{i} \mathrm{~d} s
\end{aligned}
$$




$$
\begin{aligned}
& +\frac{\left|\Lambda_{3}\right|+\left|\lambda_{1}+\lambda_{2}\right|\left|\lambda_{3}\right|\left|\lambda_{1}+\lambda_{2}-\lambda_{3}\right| t}{\left|\lambda_{1}+\lambda_{2}-\lambda_{3}\right||\Lambda|} \int_{0}^{1} \int_{0}^{s} \frac{(s-\tau)^{\vartheta-1}}{\Gamma(\vartheta)} L_{i} \mathrm{~d} \tau \mathrm{d} s \\
\leq & L_{i}\left[\frac{1}{\Gamma(\vartheta+1)}+\frac{\left|\Lambda_{1}\right|+\left|\lambda_{2}\right|\left|\lambda_{3}\right|\left|\lambda_{1}+\lambda_{2}-\lambda_{3}\right|}{\left|\lambda_{1}+\lambda_{2}-\lambda_{3}\right||\Lambda| \Gamma(\vartheta+1)}\right. \\
& \left.+\frac{\left|\Lambda_{2}\right|+\left|\lambda_{2}\right|\left(\lambda_{1}+\lambda_{2}-\lambda_{3}\right)^{2}}{\left|\lambda_{1}+\lambda_{2}-\lambda_{3}\right||\Lambda| \Gamma(\vartheta)}+\frac{\left|\Lambda_{3}\right|+\left|\lambda_{1}+\lambda_{2}\right|\left|\lambda_{3}\right|\left|\lambda_{1}+\lambda_{2}-\lambda_{3}\right|}{\left|\lambda_{1}+\lambda_{2}-\lambda_{3}\right||\Lambda| \Gamma(\vartheta+2)}\right]=L_{i} \mathcal{K}_{0}^{*}
\end{aligned}
$$

for each $t \in[0,1]$, where $\mathcal{K}_{0}^{*}$ is given in (7). Also, we have

$$
\begin{aligned}
\left|\Upsilon_{i}^{\prime} u(t)\right| \leq & \int_{0}^{t} \frac{(t-s)^{\vartheta-2}}{\Gamma(\vartheta-1)}\left|h_{i}\left(s, u_{i}(s), u_{i}^{\prime}(s)\right)\right| \mathrm{d} s \\
& +\frac{\left|\lambda_{2}\right|\left|\lambda_{3}\right|\left|\lambda_{1}+\lambda_{2}-\lambda_{3}\right|}{\left|\lambda_{1}+\lambda_{2}-\lambda_{3}\right||\Lambda|} \int_{0}^{1} \frac{(1-s)^{\vartheta-1}}{\Gamma(\vartheta)}\left|h_{i}\left(s, u_{i}(s), u_{i}^{\prime}(s)\right)\right| \mathrm{d} s \\
& +\frac{+\left|\lambda_{2}\right|\left(\lambda_{1}+\lambda_{2}-\lambda_{3}\right)^{2}}{\left|\lambda_{1}+\lambda_{2}-\lambda_{3}\right||\Lambda|} \int_{0}^{1} \frac{(1-s)^{\vartheta-2}}{\Gamma(\vartheta-1)}\left|h_{i}\left(s, u_{i}(s), u_{i}^{\prime}(s)\right)\right| \mathrm{d} s \\
& +\frac{\left|\lambda_{1}+\lambda_{2}\right|\left|\lambda_{3}\right|\left|\lambda_{1}+\lambda_{2}-\lambda_{3}\right|}{\left|\lambda_{1}+\lambda_{2}-\lambda_{3}\right||\Lambda|} \int_{0}^{1} \int_{0}^{s} \frac{(s-\tau)^{\vartheta-1}}{\Gamma(\vartheta)}\left|h_{i}\left(\tau, u_{i}(\tau), u_{i}^{\prime}(\tau)\right)\right| \mathrm{d} \tau \mathrm{d} s \\
\leq & L_{i}\left[\frac{1}{\Gamma(\vartheta)}+\frac{\left|\lambda_{2}\right|\left|\lambda_{3}\right|\left|\lambda_{1}+\lambda_{2}-\lambda_{3}\right|}{\left|\lambda_{1}+\lambda_{2}-\lambda_{3}\right||\Lambda| \Gamma(\vartheta+1)}\right. \\
& \left.+\frac{\left|\lambda_{2}\right|\left(\lambda_{1}+\lambda_{2}-\lambda_{3}\right)^{2}}{\left|\lambda_{1}+\lambda_{2}-\lambda_{3}\right||\Lambda| \Gamma(\vartheta)}+\frac{\left|\lambda_{1}+\lambda_{2}\right|\left|\lambda_{3}\right|\left|\lambda_{1}+\lambda_{2}-\lambda_{3}\right|}{\left|\lambda_{1}+\lambda_{2}-\lambda_{3}\right||\Lambda| \Gamma(\vartheta+2)}\right]=L_{i} \mathcal{K}_{1}^{*}
\end{aligned}
$$

for all $t \in[0,1]$, where $\mathcal{K}_{1}^{*}$ is given in (7). This implies that $\left\|\Upsilon_{i} u(t)\right\|_{\mathcal{X}_{i}} \leq L_{i}\left(\mathcal{K}_{0}^{*}+\mathcal{K}_{1}^{*}\right)$. Hence, $\|\Upsilon u(t)\|_{\mathcal{X}}=\sum_{i=1}^{7}\left\|\Upsilon_{i} u(t)\right\|_{\mathcal{X}_{i}} \leq \sum_{i=1}^{7} L_{i}\left(\mathcal{K}_{0}^{*}+\mathcal{K}_{1}^{*}\right)<\infty$. This shows that the operator $\Upsilon$ is uniformly bounded. Now, we prove that the operator $\Upsilon$ is equicontinuous. Now, let $u=$ $\left(u_{1}, u_{2}, \ldots, u_{7}\right) \in M$ and $t_{1}, t_{2} \in[0,1]$ with $t_{1}<t_{2}$. Then we have

$$
\begin{aligned}
\left|\Upsilon_{i} u\left(t_{2}\right)-\Upsilon_{i} u\left(t_{1}\right)\right| \leq & \int_{0}^{t_{1}} \frac{\left[\left(t_{2}-s\right)^{\vartheta-1}-\left(t_{1}-s\right)^{\vartheta-1}\right]}{\Gamma(\vartheta)}\left|h_{i}\left(s, u_{i}(s), u_{i}^{\prime}(s)\right)\right| \mathrm{d} s \\
& +\int_{t_{1}}^{t_{2}} \frac{\left(t_{2}-s\right)^{\vartheta-1}}{\Gamma(\vartheta)}\left|h_{i}\left(s, u_{i}(s), u_{i}^{\prime}(s)\right)\right| \mathrm{d} s \\
& +\frac{\left|\lambda_{2}\right|\left|\lambda_{3}\right|\left|\lambda_{1}+\lambda_{2}-\lambda_{3}\right|\left(t_{2}-t_{1}\right)}{\left|\lambda_{1}+\lambda_{2}-\lambda_{3}\right||\Lambda|} \int_{0}^{1} \frac{(1-s)^{\vartheta-1}}{\Gamma(\vartheta)}\left|h_{i}\left(s, u_{i}(s), u_{i}^{\prime}(s)\right)\right| \mathrm{d} s \\
& +\frac{\left|\lambda_{2}\right|\left(\lambda_{1}+\lambda_{2}-\lambda_{3}\right)^{2}\left(t_{2}-t_{1}\right)}{\left|\lambda_{1}+\lambda_{2}-\lambda_{3}\right||\Lambda|} \int_{0}^{1} \frac{(1-s)^{\vartheta-2}}{\Gamma(\vartheta-1)}\left|h_{i}\left(s, u_{i}(s), u_{i}^{\prime}(s)\right)\right| \mathrm{d} s \\
& +\frac{\left|\lambda_{1}+\lambda_{2}\right|\left|\lambda_{3}\right|\left|\lambda_{1}+\lambda_{2}-\lambda_{3}\right|\left(t_{2}-t_{1}\right)}{\left|\lambda_{1}+\lambda_{2}-\lambda_{3}\right||\Lambda|} \\
& \times \int_{0}^{1} \int_{0}^{s} \frac{(s-\tau)^{\vartheta-1}}{\Gamma(\vartheta)}\left|h_{i}\left(\tau, u_{i}(\tau), u_{i}^{\prime}(\tau)\right)\right| \mathrm{d} \tau \mathrm{d} s .
\end{aligned}
$$

The right-hand side of the inequality converges to zero independently of $u \in M$ as $t_{1} \rightarrow t_{2}$. Similarly, we have

$$
\begin{aligned}
\left|\Upsilon_{i}^{\prime} u\left(t_{2}\right)-\Upsilon_{i}^{\prime} u\left(t_{1}\right)\right| \leq & \int_{0}^{t_{1}} \frac{\left[\left(t_{2}-s\right)^{\vartheta-2}-\left(t_{1}-s\right)^{\vartheta-2}\right]}{\Gamma(\vartheta-1)}\left|h_{i}\left(s, u_{i}(s), u_{i}^{\prime}(s)\right)\right| \mathrm{d} s \\
& +\int_{t_{1}}^{t_{2}} \frac{\left(t_{2}-s\right)^{\vartheta-2}}{\Gamma(\vartheta-1)}\left|h_{i}\left(s, u_{i}(s), u_{i}^{\prime}(s)\right)\right| \mathrm{d} s .
\end{aligned}
$$


Again, the right-hand side of the inequality converges to zero independently of $u \in M$ as $t_{1} \rightarrow t_{2}$. Hence, $\left\|\Upsilon u\left(t_{2}\right)-\Upsilon u\left(t_{1}\right)\right\|_{\mathcal{X}} \rightarrow 0$ as $t_{1} \rightarrow t_{2}$. This shows that $\Upsilon$ is an equicontinuous operator on the product space $\mathcal{X}$. Now by using the Arzela-Ascoli theorem, one can conclude that $\Upsilon$ is a completely continuous operator. Here, consider the subset

$$
\Omega:=\left\{\left(u_{1}, u_{2}, \ldots, u_{7}\right) \in \mathcal{X}:\left(u_{1}, u_{2}, \ldots, u_{7}\right)=\mu \Upsilon\left(u_{1}, u_{2}, \ldots, u_{7}\right), \mu \in(0,1)\right\}
$$

of $\mathcal{X}$. We prove that $\Omega$ is a bounded set. Let $\left(u_{1}, u_{2}, \ldots, u_{7}\right) \in \Omega$. Then

$$
\left(u_{1}, u_{2}, \ldots, u_{7}\right)=\mu \Upsilon\left(u_{1}, u_{2}, \ldots, u_{7}\right)
$$

and so $u_{i}(t)=\mu \Upsilon_{i}\left(u_{1}, u_{2}, \ldots, u_{7}\right)$ for all $t \in[0,1]$ and $i=1,2, \ldots, 7$. Thus,

$$
\begin{aligned}
\left|u_{i}(t)\right| \leq & \mu\left[\int_{0}^{t} \frac{(t-s)^{\vartheta-1}}{\Gamma(\vartheta)}\left|h_{i}\left(s, u_{i}(s), u_{i}^{\prime}(s)\right)\right| \mathrm{d} s\right. \\
& +\frac{\left|\Lambda_{1}\right|+\left|\lambda_{2}\right|\left|\lambda_{3}\right|\left|\lambda_{1}+\lambda_{2}-\lambda_{3}\right| t}{\left|\lambda_{1}+\lambda_{2}-\lambda_{3}\right||\Lambda|} \int_{0}^{1} \frac{(1-s)^{\vartheta-1}}{\Gamma(\vartheta)}\left|h_{i}\left(s, u_{i}(s), u_{i}^{\prime}(s)\right)\right| \mathrm{d} s \\
& +\frac{\left|\Lambda_{2}\right|+\left|\lambda_{2}\right|\left(\lambda_{1}+\lambda_{2}-\lambda_{3}\right)^{2} t}{\left|\lambda_{1}+\lambda_{2}-\lambda_{3}\right||\Lambda|} \int_{0}^{1} \frac{(1-s)^{\vartheta-2}}{\Gamma(\vartheta-1)}\left|h_{i}\left(s, u_{i}(s), u_{i}^{\prime}(s)\right)\right| \mathrm{d} s \\
& +\frac{\left|\Lambda_{3}\right|+\left|\lambda_{1}+\lambda_{2}\right|\left|\lambda_{3}\right|\left|\lambda_{1}+\lambda_{2}-\lambda_{3}\right| t}{\left|\lambda_{1}+\lambda_{2}-\lambda_{3}\right||\Lambda|} \\
& \left.\times \int_{0}^{1} \int_{0}^{s} \frac{(s-\tau)^{\vartheta-1}}{\Gamma(\vartheta)}\left|h_{i}\left(\tau, u_{i}(\tau), u_{i}^{\prime}(\tau)\right)\right| \mathrm{d} \tau \mathrm{d} s\right] \\
\leq & \mu L_{i}\left[\frac{1}{\Gamma(\vartheta+1)}+\frac{\left|\Lambda_{1}\right|+\left|\lambda_{2}\right|\left|\lambda_{3}\right|\left|\lambda_{1}+\lambda_{2}-\lambda_{3}\right|}{\left|\lambda_{1}+\lambda_{2}-\lambda_{3}\right||\Lambda| \Gamma(\vartheta+1)}\right. \\
& \left.+\frac{\left|\Lambda_{2}\right|+\left|\lambda_{2}\right|\left(\lambda_{1}+\lambda_{2}-\lambda_{3}\right)^{2}}{\left|\lambda_{1}+\lambda_{2}-\lambda_{3}\right||\Lambda| \Gamma(\vartheta)}+\frac{\left|\Lambda_{3}\right|+\left|\lambda_{1}+\lambda_{2}\right|\left|\lambda_{3}\right|\left|\lambda_{1}+\lambda_{2}-\lambda_{3}\right|}{\left|\lambda_{1}+\lambda_{2}-\lambda_{3}\right||\Lambda| \Gamma(\vartheta+2)}\right]=\mu L_{i} \mathcal{K}_{0}^{*}
\end{aligned}
$$

and

$$
\begin{aligned}
\left|u_{i}^{\prime}(t)\right| \leq & \mu\left[\int_{0}^{t} \frac{(t-s)^{\vartheta-2}}{\Gamma(\vartheta-1)}\left|h_{i}\left(s, u_{i}(s), u_{i}^{\prime}(s)\right)\right| \mathrm{d} s\right. \\
& +\frac{\left|\lambda_{2}\right|\left|\lambda_{3}\right|\left|\lambda_{1}+\lambda_{2}-\lambda_{3}\right|}{\left|\lambda_{1}+\lambda_{2}-\lambda_{3}\right||\Lambda|} \int_{0}^{1} \frac{(1-s)^{\vartheta-1}}{\Gamma(\vartheta)}\left|h_{i}\left(s, u_{i}(s), u_{i}^{\prime}(s)\right)\right| \mathrm{d} s \\
& +\frac{+\left|\lambda_{2}\right|\left(\lambda_{1}+\lambda_{2}-\lambda_{3}\right)^{2}}{\left|\lambda_{1}+\lambda_{2}-\lambda_{3}\right||\Lambda|} \int_{0}^{1} \frac{(1-s)^{\vartheta-2}}{\Gamma(\vartheta-1)}\left|h_{i}\left(s, u_{i}(s), u_{i}^{\prime}(s)\right)\right| \mathrm{d} s \\
& \left.+\frac{\left|\lambda_{1}+\lambda_{2}\right|\left|\lambda_{3}\right|\left|\lambda_{1}+\lambda_{2}-\lambda_{3}\right|}{\left|\lambda_{1}+\lambda_{2}-\lambda_{3}\right||\Lambda|} \int_{0}^{1} \int_{0}^{s} \frac{(s-\tau)^{\vartheta-1}}{\Gamma(\vartheta)}\left|h_{i}\left(\tau, u_{i}(\tau), u_{i}^{\prime}(\tau)\right)\right| \mathrm{d} \tau \mathrm{d} s\right] \\
\leq & \mu L_{i}\left[\frac{1}{\Gamma(\vartheta)}+\frac{\left|\lambda_{2}\right|\left|\lambda_{3}\right|\left|\lambda_{1}+\lambda_{2}-\lambda_{3}\right|}{\left|\lambda_{1}+\lambda_{2}-\lambda_{3}\right||\Lambda| \Gamma(\vartheta+1)}\right. \\
& \left.+\frac{\left|\lambda_{2}\right|\left(\lambda_{1}+\lambda_{2}-\lambda_{3}\right)^{2}}{\left|\lambda_{1}+\lambda_{2}-\lambda_{3}\right||\Lambda| \Gamma(\vartheta)}+\frac{\left|\lambda_{1}+\lambda_{2}\right|\left|\lambda_{3}\right|\left|\lambda_{1}+\lambda_{2}-\lambda_{3}\right|}{\left|\lambda_{1}+\lambda_{2}-\lambda_{3}\right||\Lambda| \Gamma(\vartheta+2)}\right]=\mu L_{i} \mathcal{K}_{1}^{*} .
\end{aligned}
$$

This implies that $\|u\|_{\mathcal{X}}=\sum_{i=1}^{7}\left\|u_{i}\right\|_{\mathcal{X}_{i}} \leq \mu \sum_{i=1}^{7} L_{i}\left(\mathcal{K}_{0}^{*}+\mathcal{K}_{1}^{*}\right)<\infty$ and so $\Omega$ is bounded. Now, by using Lemmas 1 and 3, the operator $\Upsilon$ has a fixed point in $\mathcal{X}$ which is a solution for the fractional boundary value problem (2). 
Now, we review the fractional boundary value problem (2) under different conditions.

Theorem 5 Assume that $h_{1}, \ldots, h_{7}:[0,1] \times \mathbb{R} \times \mathbb{R} \rightarrow \mathbb{R}$ are continuous functions, there exist continuous functions $\sigma_{1}, \ldots, \sigma_{7}:[0,1] \rightarrow \mathbb{R}, \delta_{1}, \ldots, \delta_{7}:[0,1] \rightarrow \mathbb{R}^{+}$and nondecreasing continuous functions $\phi_{1}, \ldots, \phi_{7}:[0,1] \rightarrow \mathbb{R}^{+}$such that $\left|h_{i}\left(t, u_{1}, u_{2}\right)\right| \leq \delta_{i}(t) \phi_{i}\left(\left|u_{1}\right|+\left|u_{2}\right|\right)$ and

$$
\left|h_{i}\left(t, u_{1}, u_{2}\right)-h_{i}\left(t, v_{1}, v_{2}\right)\right| \leq \sigma_{i}(t)\left(\left|u_{1}-v_{1}\right|+\left|u_{2}-v_{2}\right|\right)
$$

for all $t \in[0,1], u_{1}, u_{2}, v_{1} . v_{2} \in \mathbb{R}$ and $i=1, \ldots, 7$. If $k:=\left(\Delta_{1}^{*}+\Delta_{2}^{*}\right) \sum_{i=1}^{7}\left\|\sigma_{i}\right\|<1$, then the fractional boundary value problem (2) has a solution, where $\left\|\sigma_{i}\right\|=\sup _{t \in[0,1]}\left|\sigma_{i}(t)\right|$ and the constants $\Delta_{1}^{*}$ and $\Delta_{2}^{*}$ are given in (7).

Proof Put $\left\|\delta_{i}\right\|=\sup _{t \in[0,1]}\left|\delta_{i}(t)\right|$ and choose suitable constants $\rho_{i}$ such that

$$
\rho_{i} \geq \sum_{i=1}^{7} \phi_{i}\left(\left\|u_{i}\right\| \mathcal{X}_{i}\right)\left\|\delta_{i}\right\|\left\{\mathcal{K}_{0}^{*}+\mathcal{K}_{1}^{*}\right\}
$$

where $\mathcal{K}_{i}^{*}$ s are given in (7). Consider the sets $\mathcal{B}_{\rho_{i}}:=\left\{u=\left(u_{1}, u_{2}, \ldots, u_{7}\right) \in \mathcal{X}:\|u\|_{\mathcal{X}} \leq \rho_{i}\right\}$, where $\rho_{i}$ is given in (8). It is clear that $\mathcal{B}_{\rho_{i}}$ is a closed, bounded, convex, and nonempty subset of the product Banach space $\mathcal{X}$. Now, define the operators $\Upsilon_{1}$ and $\Upsilon_{2}$ on $\mathcal{B}_{\rho_{i}}$ by

$$
\begin{aligned}
& \Upsilon_{1}\left(u_{1}, u_{2}, \ldots, u_{7}\right)(t):=\left(\Upsilon_{1}^{(1)}\left(u_{1}, u_{2}, \ldots, u_{7}\right)(t), \ldots, \Upsilon_{1}^{(7)}\left(u_{1}, u_{2}, \ldots, u_{7}\right)(t)\right) \\
& \Upsilon_{2}\left(u_{1}, u_{2}, \ldots, u_{7}\right)(t):=\left(\Upsilon_{2}^{(1)}\left(u_{1}, u_{2}, \ldots, u_{7}\right)(t), \ldots, \Upsilon_{2}^{(7)}\left(u_{1}, u_{2}, \ldots, u_{7}\right)(t)\right)
\end{aligned}
$$

where $\left(\Upsilon_{1}^{(i)} u\right)(t)=\int_{0}^{t} \frac{(t-s)^{\vartheta-1}}{\Gamma(\vartheta)} h_{i}\left(s, u_{i}(s), u_{i}^{\prime}(s)\right) \mathrm{d} s$ and

$$
\begin{aligned}
& \left(\Upsilon_{2}^{(i)} u\right)(t) \\
& =\frac{\Lambda_{1}-\lambda_{2} \lambda_{3}\left(\lambda_{1}+\lambda_{2}-\lambda_{3}\right) t}{\left(\lambda_{1}+\lambda_{2}-\lambda_{3}\right) \Lambda} \int_{0}^{1} \frac{(1-s)^{\vartheta-1}}{\Gamma(\vartheta)} h_{i}\left(s, u_{i}(s), u_{i}^{\prime}(s)\right) \mathrm{d} s \\
& \quad+\frac{\Lambda_{2}-\lambda_{2}\left(\lambda_{1}+\lambda_{2}-\lambda_{3}\right)^{2} t}{\left(\lambda_{1}+\lambda_{2}-\lambda_{3}\right) \Lambda} \int_{0}^{1} \frac{(1-s)^{\vartheta-2}}{\Gamma(\vartheta-1)} h_{i}\left(s, u_{i}(s), u_{i}^{\prime}(s)\right) \mathrm{d} s \\
& \quad+\frac{\Lambda_{3}+\left(\lambda_{1}+\lambda_{2}\right) \lambda_{3}\left(\lambda_{1}+\lambda_{2}-\lambda_{3}\right) t}{\left(\lambda_{1}+\lambda_{2}-\lambda_{3}\right) \Lambda} \int_{0}^{1} \int_{0}^{s} \frac{(s-\tau)^{\vartheta-1}}{\Gamma(\vartheta)} h_{i}\left(\tau, u_{i}(\tau), u_{i}^{\prime}(\tau)\right) \mathrm{d} \tau \mathrm{d} s
\end{aligned}
$$

for all $t \in[0,1]$ and $u=\left(u_{1}, u_{2}, \ldots, u_{7}\right) \in \mathcal{B}_{\rho_{i}}$. Let $\phi_{i}^{*}=\sup _{u_{i} \in \mathcal{X}_{i}} \phi_{i}\left(\left\|u_{i}\right\|_{\mathcal{X}_{i}}\right)$. Now, for every $u=\left(u_{1}, \ldots, u_{7}\right), v=\left(v_{1}, \ldots, v_{7}\right) \in \mathcal{B}_{\rho_{i}}$, we have

$$
\begin{aligned}
& \left|\left(\Upsilon_{1}^{(i)} u+\Upsilon_{2}^{(i)} v\right)(t)\right| \\
& \quad \leq \int_{0}^{t} \frac{(t-s)^{\vartheta-1}}{\Gamma(\vartheta)}\left|h_{i}\left(s, u_{i}(s), u_{i}^{\prime}(s)\right)\right| \mathrm{d} s \\
& \quad+\frac{\left|\Lambda_{1}\right|+\left|\lambda_{2}\right|\left|\lambda_{3}\right|\left|\lambda_{1}+\lambda_{2}-\lambda_{3}\right| t}{\left|\lambda_{1}+\lambda_{2}-\lambda_{3}\right||\Lambda|} \int_{0}^{1} \frac{(1-s)^{\vartheta-1}}{\Gamma(\vartheta)}\left|h_{i}\left(s, v_{i}(s), v_{i}^{\prime}(s)\right)\right| \mathrm{d} s \\
& \quad+\frac{\left|\Lambda_{2}\right|+\left|\lambda_{2}\right|\left(\lambda_{1}+\lambda_{2}-\lambda_{3}\right)^{2} t}{\left|\lambda_{1}+\lambda_{2}-\lambda_{3}\right||\Lambda|} \int_{0}^{1} \frac{(1-s)^{\vartheta-2}}{\Gamma(\vartheta-1)}\left|h_{i}\left(s, v_{i}(s), v_{i}^{\prime}(s)\right)\right| \mathrm{d} s
\end{aligned}
$$




$$
\begin{aligned}
& +\frac{\left|\Lambda_{3}\right|+\left|\lambda_{1}+\lambda_{2}\right|\left|\lambda_{3}\right|\left|\lambda_{1}+\lambda_{2}-\lambda_{3}\right| t}{\left|\lambda_{1}+\lambda_{2}-\lambda_{3}\right||\Lambda|} \\
& \times \int_{0}^{1} \int_{0}^{s} \frac{(s-\tau)^{\vartheta-1}}{\Gamma(\vartheta)}\left|h_{i}\left(\tau, v_{i}(\tau), v_{i}^{\prime}(\tau)\right)\right| \mathrm{d} \tau \mathrm{d} s \\
\leq & \int_{0}^{t} \frac{(t-s)^{\vartheta-1}}{\Gamma(\vartheta)} \delta_{i}(s) \phi_{i}\left(\left|u_{i}(s)\right|+\left|u_{i}^{\prime}(s)\right|\right) \mathrm{d} s \\
& +\frac{\left|\Lambda_{1}\right|+\left|\lambda_{2}\right|\left|\lambda_{3}\right|\left|\lambda_{1}+\lambda_{2}-\lambda_{3}\right| t}{\left|\lambda_{1}+\lambda_{2}-\lambda_{3}\right||\Lambda|} \int_{0}^{1} \frac{(1-s)^{\vartheta-1}}{\Gamma(\vartheta)} \delta_{i}(s) \phi_{i}\left(\left|v_{i}(s)\right|+\left|v_{i}^{\prime}(s)\right|\right) \mathrm{d} s \\
& +\frac{\left|\Lambda_{2}\right|+\left|\lambda_{2}\right|\left(\lambda_{1}+\lambda_{2}-\lambda_{3}\right)^{2} t}{\left|\lambda_{1}+\lambda_{2}-\lambda_{3}\right||\Lambda|} \int_{0}^{1} \frac{(1-s)^{\vartheta-2}}{\Gamma(\vartheta-1)} \delta_{i}(s) \phi_{i}\left(\left|v_{i}(s)\right|+\left|v_{i}^{\prime}(s)\right|\right) \mathrm{d} s \\
& +\frac{\left|\Lambda_{3}\right|+\left|\lambda_{1}+\lambda_{2}\right|\left|\lambda_{3}\right|\left|\lambda_{1}+\lambda_{2}-\lambda_{3}\right| t}{\left|\lambda_{1}+\lambda_{2}-\lambda_{3}\right||\Lambda|} \\
& \times \int_{0}^{1} \int_{0}^{s} \frac{(s-\tau)^{\vartheta-1}}{\Gamma(\vartheta)} \delta_{i}(\tau) \phi_{i}\left(\left|v_{i}(\tau)\right|+\left|v_{i}^{\prime}(\tau)\right|\right) \mathrm{d} \tau \mathrm{d} s \\
\leq & \left\|\delta_{i}\right\| \phi_{i}^{*}\left[\frac{1}{\Gamma(\vartheta+1)}+\frac{\left|\Lambda_{1}\right|+\left|\lambda_{2}\right|\left|\lambda_{3}\right|\left|\lambda_{1}+\lambda_{2}-\lambda_{3}\right|}{\left|\lambda_{1}+\lambda_{2}-\lambda_{3}\right||\Lambda| \Gamma(\vartheta+1)}\right. \\
& \left.+\frac{\left|\Lambda_{2}\right|+\left|\lambda_{2}\right|\left(\lambda_{1}+\lambda_{2}-\lambda_{3}\right)^{2}}{\left|\lambda_{1}+\lambda_{2}-\lambda_{3}\right||\Lambda| \Gamma(\vartheta)}+\frac{\left|\Lambda_{3}\right|+\left|\lambda_{1}+\lambda_{2}\right|\left|\lambda_{3}\right|\left|\lambda_{1}+\lambda_{2}-\lambda_{3}\right|}{\left|\lambda_{1}+\lambda_{2}-\lambda_{3}\right||\Lambda| \Gamma(\vartheta+2)}\right]=\left\|\delta_{i}\right\| \phi_{i}^{*} \mathcal{K}_{0}^{*},
\end{aligned}
$$

and

$$
\begin{aligned}
&\left|\left(\left(Y_{1}^{(i)}\right)^{\prime} u+\left(\Upsilon_{2}^{(i)}\right)^{\prime} v\right)(t)\right| \\
& \leq \int_{0}^{t} \frac{(t-s)^{\vartheta-2}}{\Gamma(\vartheta-1)}\left|h_{i}\left(s, u_{i}(s), u_{i}^{\prime}(s)\right)\right| \mathrm{d} s \\
& \quad+\frac{\left|\lambda_{2}\right|\left|\lambda_{3}\right|\left|\lambda_{1}+\lambda_{2}-\lambda_{3}\right|}{\left|\lambda_{1}+\lambda_{2}-\lambda_{3}\right||\Lambda|} \int_{0}^{1} \frac{(1-s)^{\vartheta-1}}{\Gamma(\vartheta)}\left|h_{i}\left(s, v_{i}(s), v_{i}^{\prime}(s)\right)\right| \mathrm{d} s \\
& \quad+\frac{\left|\lambda_{2}\right|\left(\lambda_{1}+\lambda_{2}-\lambda_{3}\right)^{2}}{\left|\lambda_{1}+\lambda_{2}-\lambda_{3}\right||\Lambda|} \int_{0}^{1} \frac{(1-s)^{\vartheta-2}}{\Gamma(\vartheta-1)}\left|h_{i}\left(s, v_{i}(s), v_{i}^{\prime}(s)\right)\right| \mathrm{d} s \\
& \quad+\frac{\left|\lambda_{1}+\lambda_{2}\right|\left|\lambda_{3}\right|\left|\lambda_{1}+\lambda_{2}-\lambda_{3}\right|}{\left|\lambda_{1}+\lambda_{2}-\lambda_{3}\right||\Lambda|} \int_{0}^{1} \int_{0}^{s} \frac{(s-\tau)^{\vartheta-1}}{\Gamma(\vartheta)}\left|h_{i}\left(\tau, v_{i}(\tau), v_{i}^{\prime}(\tau)\right)\right| \mathrm{d} \tau \mathrm{d} s \\
& \leq \int_{0}^{t} \frac{(t-s)^{\vartheta-2}}{\Gamma(\vartheta-1)} \delta_{i}(s) \phi_{i}\left(\left|u_{i}(s)\right|+\left|u_{i}^{\prime}(s)\right|\right) \mathrm{d} s \\
&+\frac{\left|\lambda_{2}\right|\left|\lambda_{3}\right|\left|\lambda_{1}+\lambda_{2}-\lambda_{3}\right|}{\left|\lambda_{1}+\lambda_{2}-\lambda_{3}\right||\Lambda|} \int_{0}^{1} \frac{(1-s)^{\vartheta-1}}{\Gamma(\vartheta)} \delta_{i}(s) \phi_{i}\left(\left|v_{i}(s)\right|+\left|v_{i}^{\prime}(s)\right|\right) \mathrm{d} s \\
&+\frac{\left|\lambda_{2}\right|\left(\lambda_{1}+\lambda_{2}-\lambda_{3}\right)^{2}}{\left|\lambda_{1}+\lambda_{2}-\lambda_{3}\right||\Lambda|} \int_{0}^{1} \frac{(1-s)^{\vartheta-2}}{\Gamma(\vartheta-1)} \delta_{i}(s) \phi_{i}\left(\left|v_{i}(s)\right|+\left|v_{i}^{\prime}(s)\right|\right) \mathrm{d} s \\
&+\frac{\left|\lambda_{1}+\lambda_{2}\right|\left|\lambda_{3}\right|\left|\lambda_{1}+\lambda_{2}-\lambda_{3}\right|}{\left|\lambda_{1}+\lambda_{2}-\lambda_{3}\right||\Lambda|} \\
& \times \int_{0}^{1} \int_{0}^{s} \frac{(s-\tau)^{\vartheta-1}}{\Gamma(\vartheta)} \delta_{i}(\tau) \phi_{i}\left(\left|v_{i}(\tau)\right|+\left|v_{i}^{\prime}(\tau)\right|\right) \mathrm{d} \tau \mathrm{d} s \\
& \leq\left\|\delta_{i}\right\| \phi_{i}^{*}\left[\frac{1}{\Gamma(\vartheta)}+\frac{\left|\lambda_{2}\right|\left|\lambda_{3} \| \lambda_{1}+\lambda_{2}-\lambda_{3}\right|}{\left|\lambda_{1}+\lambda_{2}-\lambda_{3}\right||\Lambda| \Gamma(\vartheta+1)}+\frac{\left|\lambda_{2}\right|\left(\lambda_{1}+\lambda_{2}-\lambda_{3}\right)^{2}}{\left|\lambda_{1}+\lambda_{2}-\lambda_{3}\right||\Lambda| \Gamma(\vartheta)}\right. \\
&+\frac{\left|\lambda_{1}+\lambda_{2}\right|\left|\lambda_{3}\right|\left|\lambda_{1}+\lambda_{2}-\lambda_{3}\right|}{\left|\lambda_{1}+\lambda_{2}-\lambda_{3}\right||\Lambda| \Gamma(\vartheta+2)}=\left\|\delta_{i}\right\| \phi_{i}^{*} \mathcal{K}_{1}^{*} . \\
&
\end{aligned}
$$


This yields $\left\|\Upsilon_{1} u+\Upsilon_{2} v\right\|_{\mathcal{X}}=\sum_{i=1}^{7}\left\|\Upsilon_{1}^{(i)} u+\Upsilon_{2}^{(i)} v\right\| \mathcal{X}_{i} \leq \sum_{i=1}^{7}\left\|\delta_{i}\right\| \phi_{i}^{*}\left(\mathcal{K}_{0}^{*}+\mathcal{K}_{1}^{*}\right) \leq \rho_{i}$, and so $\left\|\Upsilon_{1} u+\Upsilon_{2} v\right\|_{\mathcal{X}} \leq \rho_{i}$ and $\Upsilon_{1} u+\Upsilon_{2} v \in \mathcal{B}_{\rho_{i}}$. On the other hand, it is clear that the continuity of $\Upsilon_{1}$ follows from the continuity of functions $h_{i}$. Now, we show that the operator $\Upsilon_{1}$ is uniformly bounded. For this, note that

$$
\begin{aligned}
\left|\left(\Upsilon_{1}^{(i)} u\right)(t)\right| & \leq \int_{0}^{t} \frac{(t-s)^{\vartheta-1}}{\Gamma(\vartheta)}\left|h_{i}\left(s, u_{i}(s), u_{i}^{\prime}(s)\right)\right| \mathrm{d} s \\
& \leq \frac{1}{\Gamma(\vartheta+1)}\left\|\delta_{i}\right\| \phi_{i}\left(\left|u_{i}(t)\right|+\left|u_{i}^{\prime}(t)\right|\right),
\end{aligned}
$$

and $\left|\left(\left(\Upsilon_{1}^{(i)}\right)^{\prime} u\right)(t)\right| \leq \int_{0}^{t} \frac{\left(t-s \vartheta^{\vartheta-2}\right.}{\Gamma(\vartheta-1)}\left|h_{i}\left(s, u_{i}(s), u_{i}^{\prime}(s)\right)\right| \mathrm{d} s \leq \frac{1}{\Gamma(\vartheta)}\left\|\delta_{i}\right\| \phi_{i}\left(\left|u_{i}(t)\right|+\left|u_{i}^{\prime}(t)\right|\right)$ for all $u$ in $\mathcal{B}_{\rho_{i}}$. Thus, $\left\|\Upsilon_{1} u\right\|_{\mathcal{X}}=\sum_{i=1}^{7}\left\|\Upsilon_{1}^{(i)} u\right\|_{\mathcal{X}_{i}} \leq\left\{\frac{1}{\Gamma(\vartheta+1)}+\frac{1}{\Gamma(\vartheta)}\right\} \sum_{i=1}^{7}\left\|\delta_{i}\right\| \phi_{i}\left(\left\|u_{i}\right\|_{\mathcal{X}_{i}}\right)$. This shows that the operator $\Upsilon_{1}$ is uniformly bounded on $\mathcal{B}_{\rho_{i}}$. Now, we show that the operator $\Upsilon_{1}$ is compact on $\mathcal{B}_{\rho_{i}}$. Let $t_{1}, t_{2} \in[0,1]$ with $t_{1}<t_{2}$. Then we have

$$
\begin{aligned}
\left|\left(\Upsilon_{1}^{(i)} u\right)\left(t_{2}\right)-\left(\Upsilon_{1}^{(i)} u\right)\left(t_{1}\right)\right| & \\
= & \mid \int_{0}^{t_{2}} \frac{\left(t_{2}-s\right)^{\vartheta-1}}{\Gamma(\vartheta)} h_{i}\left(s, u_{i}(s), u_{i}^{\prime}(s)\right) \mathrm{d} s \\
& -\int_{0}^{t_{1}} \frac{\left(t_{1}-s\right)^{\vartheta-1}}{\Gamma(\vartheta)} h_{i}\left(s, u_{i}(s), u_{i}^{\prime}(s)\right) \mathrm{d} s \mid \\
\leq & \left|\int_{0}^{t_{1}} \frac{\left[\left(t_{2}-s\right)^{(\vartheta-1)}-\left(t_{1}-s\right)^{(\vartheta-1)}\right]}{\Gamma(\vartheta)} h_{i}\left(s, u_{i}(s), u_{i}^{\prime}(s)\right) \mathrm{d} s\right| \\
& +\left|\int_{t_{1}}^{t_{2}} \frac{\left(t_{2}-s\right)^{(\vartheta-1)}}{\Gamma(\vartheta)} h_{i}\left(s, u_{i}(s), u_{i}^{\prime}(s)\right) \mathrm{d} s\right| \\
\leq & \int_{0}^{t_{1}} \frac{\left[\left(t_{2}-s\right)^{(\vartheta-1)}-\left(t_{1}-s\right)^{(\vartheta-1)}\right]}{\Gamma(\vartheta)}\left|h_{i}\left(s, u_{i}(s), u_{i}^{\prime}(s)\right)\right| \mathrm{d} s \\
& +\int_{t_{1}}^{t_{2}} \frac{\left(t_{2}-s\right)^{(\vartheta-1)}}{\Gamma(\vartheta)}\left|h_{i}\left(s, u_{i}(s), u_{i}^{\prime}(s)\right)\right| \mathrm{d} s \\
\leq & \left\{\frac{t_{2}^{\vartheta}-t_{1}^{\vartheta}-\left(t_{2}-t_{1}\right)^{\vartheta}}{\Gamma(\vartheta+1)}+\frac{\left(t_{2}-t_{1}\right)^{\vartheta}}{\Gamma(\vartheta+1)}\right\}\left\|\delta_{i}\right\| \phi_{i}\left(\left\|u_{i}\right\| \mathcal{X}_{i}\right) .
\end{aligned}
$$

Hence, $\left|\left(\Upsilon_{1}^{(i)} u\right)\left(t_{2}\right)-\left(\Upsilon_{1}^{(i)} u\right)\left(t_{1}\right)\right| \rightarrow 0$ as $t_{1} \rightarrow t_{2}$. Also, we have

$$
\begin{aligned}
& \left|\left(\left(\Upsilon_{1}^{(i)}\right)^{\prime} u\right)\left(t_{2}\right)-\left(\left(\Upsilon_{1}^{(i)}\right)^{\prime} u\right)\left(t_{1}\right)\right| \\
& =\mid \int_{0}^{t_{2}} \frac{\left(t_{2}-s\right)^{\vartheta-2}}{\Gamma(\vartheta-1)} h_{i}\left(s, u_{i}(s), u_{i}^{\prime}(s)\right) \mathrm{d} s \\
& \quad-\int_{0}^{t_{1}} \frac{\left(t_{1}-s\right)^{\vartheta-2}}{\Gamma(\vartheta-1)} h_{i}\left(s, u_{i}(s), u_{i}^{\prime}(s)\right) \mathrm{d} s \mid \\
& \leq\left|\int_{0}^{t_{1}} \frac{\left[\left(t_{2}-s\right)^{(\vartheta-2)}-\left(t_{1}-s\right)^{(\vartheta-2)}\right]}{\Gamma(\vartheta-1)} h_{i}\left(s, u_{i}(s), u_{i}^{\prime}(s)\right) \mathrm{d} s\right| \\
& \quad+\left|\int_{t_{1}}^{t_{2}} \frac{\left(t_{2}-s\right)^{(\vartheta-2)}}{\Gamma(\vartheta-1)} h_{i}\left(s, u_{i}(s), u_{i}^{\prime}(s)\right) \mathrm{d} s\right| \\
& \leq \int_{0}^{t_{1}} \frac{\left[\left(t_{2}-s\right)^{(\vartheta-2)}-\left(t_{1}-s\right)^{(\vartheta-2)}\right]}{\Gamma(\vartheta-1)}\left|h_{i}\left(s, u_{i}(s), u_{i}^{\prime}(s)\right)\right| \mathrm{d} s
\end{aligned}
$$




$$
\begin{aligned}
& +\int_{t_{1}}^{t_{2}} \frac{\left(t_{2}-s\right)^{(\vartheta-2)}}{\Gamma(\vartheta-1)}\left|h_{i}\left(s, u_{i}(s), u_{i}^{\prime}(s)\right)\right| \mathrm{d} s \\
\leq & \left\{\frac{t_{2}^{\vartheta-1}-t_{1}^{\vartheta-1}-\left(t_{2}-t_{1}\right)^{\vartheta-1}}{\Gamma(\vartheta)}+\frac{\left(t_{2}-t_{1}\right)^{\vartheta-1}}{\Gamma(\vartheta)}\right\}\left\|\delta_{i}\right\| \phi_{i}\left(\left\|u_{i}\right\|_{\mathcal{X}_{i}}\right),
\end{aligned}
$$

and so $\left|\left(\left(\Upsilon_{1}^{(i)}\right)^{\prime} u\right)\left(t_{2}\right)-\left(\left(\Upsilon_{1}^{(i)}\right)^{\prime} u\right)\left(t_{1}\right)\right| \rightarrow 0$ as $t_{1} \rightarrow t_{2}$. Hence, $\left\|\left(\Upsilon_{1} u\right)\left(t_{2}\right)-\left(\Upsilon_{1} u\right)\left(t_{1}\right)\right\|_{\mathcal{X}}$ tends to zero as $t_{1} \rightarrow t_{2}$. Thus, $\Upsilon_{1}$ is equicontinuous, and so $\Upsilon_{1}$ is a relatively compact operator on $\mathcal{B}_{\rho_{i}}$. Now, by using the Arzela-Ascoli theorem, we conclude that the operator $\Upsilon_{1}$ is compact on $\mathcal{B}_{\rho_{i}}$. Here, we prove that the operator $\Upsilon_{2}$ is a contraction. Let $u, v \in \mathcal{B}_{\rho_{i}}$. Then we have

$$
\begin{aligned}
&\left|\left(\Upsilon_{2}^{(i)} u\right)(t)-\left(\Upsilon_{2}^{(i)} v\right)(t)\right| \\
& \leq \frac{\left|\Lambda_{1}\right|+\left|\lambda_{2}\right|\left|\lambda_{3}\right|\left|\lambda_{1}+\lambda_{2}-\lambda_{3}\right| t}{\left|\lambda_{1}+\lambda_{2}-\lambda_{3}\right||\Lambda|} \\
& \times \int_{0}^{1} \frac{(1-s)^{\vartheta-1}}{\Gamma(\vartheta)}\left|h_{i}\left(s, u_{i}(s), u_{i}^{\prime}(s)\right)-h_{i}\left(s, v_{i}(s), v_{i}^{\prime}(s)\right)\right| \mathrm{d} s \\
&+\frac{\left|\Lambda_{2}\right|+\left|\lambda_{2}\right|\left(\lambda_{1}+\lambda_{2}-\lambda_{3}\right)^{2} t}{\left|\lambda_{1}+\lambda_{2}-\lambda_{3}\right||\Lambda|} \\
& \times \int_{0}^{1} \frac{(1-s)^{\vartheta-2}}{\Gamma(\vartheta-1)}\left|h_{i}\left(s, u_{i}(s), u_{i}^{\prime}(s)\right)-h_{i}\left(s, v_{i}(s), v_{i}^{\prime}(s)\right)\right| \mathrm{d} s \\
&+\frac{\left|\Lambda_{3}\right|+\left|\lambda_{1}+\lambda_{2}\right|\left|\lambda_{3}\right|\left|\lambda_{1}+\lambda_{2}-\lambda_{3}\right| t}{\left|\lambda_{1}+\lambda_{2}-\lambda_{3}\right||\Lambda|} \\
& \times \int_{0}^{1} \int_{0}^{s} \frac{(s-\tau)^{\vartheta-1}}{\Gamma(\vartheta)}\left|h_{i}\left(\tau, u_{i}(\tau), u_{i}^{\prime}(\tau)\right)-h_{i}\left(\tau, v_{i}(\tau), v_{i}^{\prime}(\tau)\right)\right| \mathrm{d} \tau \mathrm{d} s \\
& \leq \frac{\left|\Lambda_{1}\right|+\left|\lambda_{2}\right|\left|\lambda_{3}\right|\left|\lambda_{1}+\lambda_{2}-\lambda_{3}\right| t}{\left|\lambda_{1}+\lambda_{2}-\lambda_{3}\right||\Lambda|} \\
& \times \int_{0}^{1} \frac{(1-s)^{\vartheta-1}}{\Gamma(\vartheta)} \sigma_{i}(s)\left(\left|u_{i}(s)-v_{i}(s)\right|+\left|u_{i}^{\prime}(s)-v_{i}^{\prime}(s)\right|\right) \mathrm{d} s \\
&+\frac{\left|\Lambda_{2}\right|+\left|\lambda_{2}\right|\left(\lambda_{1}+\lambda_{2}-\lambda_{3}\right)^{2} t}{\left|\lambda_{1}+\lambda_{2}-\lambda_{3}\right||\Lambda|} \\
& \times \int_{0}^{1} \frac{(1-s)^{\vartheta-2}}{\Gamma(\vartheta-1)} \sigma_{i}(s)\left(\left|u_{i}(s)-v_{i}(s)\right|+\left|u_{i}^{\prime}(s)-v_{i}^{\prime}(s)\right|\right) \mathrm{d} s \\
&+\frac{\left|\Lambda_{3}\right|+\left|\lambda_{1}+\lambda_{2}\right|\left|\lambda_{3}\right|\left|\lambda_{1}+\lambda_{2}-\lambda_{3}\right| t}{\left|\lambda_{1}+\lambda_{2}-\lambda_{3}\right||\Lambda|} \\
&=\int_{0}^{s} \frac{(s-\tau)^{\vartheta-1}}{\Gamma(\vartheta)} \sigma_{i}(\tau)\left(\left|u_{i}(\tau)-v_{i}(\tau)\right|+\left|u_{i}^{\prime}(\tau)-v_{i}^{\prime}(\tau)\right|\right) \mathrm{d} \tau \mathrm{d} s
\end{aligned}
$$

and

$$
\begin{aligned}
& \left|\left(\left(\Upsilon_{2}^{(i)}\right)^{\prime} u\right)(t)-\left(\left(\Upsilon_{2}^{(i)}\right)^{\prime} v\right)(t)\right| \\
& \quad \leq \frac{\left|\lambda_{2}\right|\left|\lambda_{3}\right|\left|\lambda_{1}+\lambda_{2}-\lambda_{3}\right|}{\left|\lambda_{1}+\lambda_{2}-\lambda_{3}\right||\Lambda|} \\
& \quad \times \int_{0}^{1} \frac{(1-s)^{\vartheta-1}}{\Gamma(\vartheta)}\left|h_{i}\left(s, u_{i}(s), u_{i}^{\prime}(s)\right)-h_{i}\left(s, v_{i}(s), v_{i}^{\prime}(s)\right)\right| \mathrm{d} s
\end{aligned}
$$




$$
\begin{aligned}
& +\frac{\left|\lambda_{2}\right|\left(\lambda_{1}+\lambda_{2}-\lambda_{3}\right)^{2}}{\left|\lambda_{1}+\lambda_{2}-\lambda_{3}\right||\Lambda|} \\
& \times \int_{0}^{1} \frac{(1-s)^{\vartheta-2}}{\Gamma(\vartheta-1)}\left|h_{i}\left(s, u_{i}(s), u_{i}^{\prime}(s)\right)-h_{i}\left(s, v_{i}(s), v_{i}^{\prime}(s)\right)\right| \mathrm{d} s \\
& +\frac{\left|\lambda_{1}+\lambda_{2}\right|\left|\lambda_{3}\right|\left|\lambda_{1}+\lambda_{2}-\lambda_{3}\right|}{\left|\lambda_{1}+\lambda_{2}-\lambda_{3}\right||\Lambda|} \\
& \times \int_{0}^{1} \int_{0}^{s} \frac{(s-\tau)^{\vartheta-1}}{\Gamma(\vartheta)}\left|h_{i}\left(\tau, u_{i}(\tau), u_{i}^{\prime}(\tau)\right)-h_{i}\left(\tau, v_{i}(\tau), v_{i}^{\prime}(\tau)\right)\right| \mathrm{d} \tau \mathrm{d} s \\
& \leq \frac{\left|\lambda_{2}\right|\left|\lambda_{3}\right|\left|\lambda_{1}+\lambda_{2}-\lambda_{3}\right|}{\left|\lambda_{1}+\lambda_{2}-\lambda_{3}\right||\Lambda|} \\
& \times \int_{0}^{1} \frac{(1-s)^{\vartheta-1}}{\Gamma(\vartheta)} \sigma_{i}(s)\left(\left|u_{i}(s)-v_{i}(s)\right|+\left|u_{i}^{\prime}(s)-v_{i}^{\prime}(s)\right|\right) \mathrm{d} s \\
& +\frac{\left|\lambda_{2}\right|\left(\lambda_{1}+\lambda_{2}-\lambda_{3}\right)^{2}}{\left|\lambda_{1}+\lambda_{2}-\lambda_{3}\right||\Lambda|} \\
& \times \int_{0}^{1} \frac{(1-s)^{\vartheta-2}}{\Gamma(\vartheta-1)} \sigma_{i}(s)\left(\left|u_{i}(s)-v_{i}(s)\right|+\left|u_{i}^{\prime}(s)-v_{i}^{\prime}(s)\right|\right) \mathrm{d} s \\
& +\frac{\left|\lambda_{1}+\lambda_{2}\right|\left|\lambda_{3}\right|\left|\lambda_{1}+\lambda_{2}-\lambda_{3}\right|}{\left|\lambda_{1}+\lambda_{2}-\lambda_{3}\right||\Lambda|} \\
& \times \int_{0}^{1} \int_{0}^{s} \frac{(s-\tau)^{\vartheta-1}}{\Gamma(\vartheta)} \sigma_{i}(\tau)\left(\left|u_{i}(\tau)-v_{i}(\tau)\right|+\left|u_{i}^{\prime}(\tau)-v_{i}^{\prime}(\tau)\right|\right) \mathrm{d} \tau \mathrm{d} s .
\end{aligned}
$$

Hence, we obtain

$$
\begin{aligned}
& \sup _{t \in[0,1]}\left|\left(\Upsilon_{2}^{(i)} u\right)(t)-\left(\Upsilon_{2}^{(i)} v\right)(t)\right| \leq\left\|\sigma_{i}\right\| \Delta_{1}^{*}\left\|u_{i}-v_{i}\right\| \mathcal{X}_{i}, \\
& \sup _{t \in[0,1]}\left|\left(\left(\Upsilon_{2}^{(i)}\right)^{\prime} u\right)(t)-\left(\left(\Upsilon_{2}^{(i)}\right)^{\prime} v\right)(t)\right| \leq\left\|\sigma_{i}\right\| \Delta_{2}^{*}\left\|u_{i}-v_{i}\right\|_{\mathcal{X}_{i}} .
\end{aligned}
$$

Thus, we get

$$
\left\|\Upsilon_{2} u-\Upsilon_{2} v\right\|_{\mathcal{X}}=\sum_{i=1}^{7}\left\|\Upsilon_{2}^{(i)} u-\Upsilon_{2}^{(i)} v\right\|_{\mathcal{X}_{i}} \leq\left(\Delta_{1}^{*}+\Delta_{2}^{*}\right) \sum_{i=1}^{7}\left\|\sigma_{i}\right\|\left\|u_{i}-v_{i}\right\|_{\mathcal{X}_{i}}
$$

and so $\left\|\Upsilon_{2} u-\Upsilon_{2} v\right\|_{\mathcal{X}} \leq k\|u-v\|_{\mathcal{X}}$. Since $k<1, \Upsilon_{2}$ is a contraction on $\mathcal{B}_{\rho_{i}}$. Now, by using Lemma 2 , we conclude that the operator $\Upsilon$ has a fixed point which is a solution for the fractional boundary value problem (2).

Finally, one can easily prove the uniqueness of solutions for the fractional problem (2).

Theorem 6 Let $h_{1}, \ldots, h_{7}:[0,1] \times \mathbb{R} \times \mathbb{R} \rightarrow \mathbb{R}$ be continuous functions. Assume that there exist constants $L_{i}>0$ such that $\left|h_{i}\left(t, u_{1}, u_{2}\right)-h_{i}\left(t, u_{1}^{\prime}, u_{2}^{\prime}\right)\right| \leq L_{i}\left(\left|u_{1}-u_{1}^{\prime}\right|+\left|u_{2}-u_{2}^{\prime}\right|\right)$ for all $u_{1}, u_{2}, u_{1}^{\prime}, u_{2}^{\prime} \in \mathbb{R}$ and $t \in[0,1](i=1,2, \ldots, 7)$. Then the fractional boundary value problem (2) has a unique solution if $\sum_{i=1}^{7} L_{i}\left(\mathcal{K}_{0}^{*}+\mathcal{K}_{1}^{*}\right)<1$.

Now, we present an example to illustrate our last main result. 
Example 1 Consider the system of fractional differential equations

$$
\left\{\begin{array}{l}
{ }^{c} \mathcal{D}_{0}^{1.08} u_{1}(t)=\frac{t\left|u_{1}(t)\right|}{10\left(1+\left|u_{1}(t)\right|\right)}+0.1 t\left|\arcsin u_{1}^{\prime}(t)\right|, \\
{ }^{c} \mathcal{D}_{0}^{1.08} u_{2}(t)=\frac{t}{2000}\left|\arctan u_{2}(t)\right|+\frac{t\left|\arcsin u_{2}^{\prime}(t)\right|}{2000+2000|| \arcsin u_{2}^{\prime}(t) \mid}, \\
{ }^{c} \mathcal{D}_{0}^{1.08} u_{3}(t)=0.01 t\left|\sin u_{3}(t)\right|+0.01 t \frac{\left|u_{3}^{\prime}(t)\right|^{3}}{2+\left|u_{3}^{\prime}(t)\right|^{3}}
\end{array}\right.
$$

with integral boundary value conditions

$$
\left\{\begin{array}{l}
0.7 u_{1}(0)+0.12 u_{1}(1)=0.1 \int_{0}^{1} u_{1}(s) \mathrm{d} s, \\
0.7 u_{1}^{\prime}(0)+0.12 u_{1}^{\prime}(1)=0.1 \int_{0}^{1} u_{1}(s) \mathrm{d} s, \\
0.7 u_{2}(0)+0.12 u_{2}(1)=0.1 \int_{0}^{1} u_{2}(s) \mathrm{d} s, \\
0.7 u_{2}^{\prime}(0)+0.12 u_{2}^{\prime}(1)=0.1 \int_{0}^{1} u_{2}(s) \mathrm{d} s, \\
0.7 u_{3}(0)+0.12 u_{3}(1)=0.1 \int_{0}^{1} u_{3}(s) \mathrm{d} s, \\
0.7 u_{3}^{\prime}(0)+0.12 u_{3}^{\prime}(1)=0.1 \int_{0}^{1} u_{3}(s) \mathrm{d} s,
\end{array}\right.
$$

where $\vartheta=1.08, \lambda_{1}=0.7, \lambda_{2}=0.12, \lambda_{3}=0.1, n=3$ and ${ }^{c} \mathcal{D}_{0}^{1.08}$ denotes the Caputo fractional derivative of order $\vartheta=1.08$. Define continuous functions $h_{1}, h_{2}, h_{3}:[0,1] \times \mathbb{R} \times \mathbb{R} \rightarrow \mathbb{R}$ by

$$
\left\{\begin{array}{l}
h_{1}(t, u(t), v(t))=\frac{t|u(t)|}{10(1+|u(t)|)}+0.1 t|\arcsin v(t)|, \\
h_{2}(t, u(t), v(t))=\frac{t}{2000}|\arctan u(t)|+\frac{t|\arcsin v(t)|}{2000+2000|| \arcsin v(t) \mid}, \\
h_{3}(t, u(t), v(t))=0.01 t|\sin u(t)|+0.01 t \frac{|v(t)|{ }^{3}}{2+|v(t)|^{3}} .
\end{array}\right.
$$

Let $u_{1}, u_{2}, v_{1}, v_{2} \in \mathbb{R}$. Then we have

$$
\begin{aligned}
& \left|h_{1}\left(t, u_{1}(t), v_{1}(t)\right)-h_{1}\left(t, u_{2}(t), v_{2}(t)\right)\right| \\
& \quad \leq \frac{t}{10}\left(\left|u_{1}(t)-u_{2}(t)\right|+\left|\arcsin v_{1}(t)-\arcsin v_{2}(t)\right|\right) \\
& \quad \leq \frac{t}{10}\left(\left|u_{1}(t)-u_{2}(t)\right|+\left|v_{1}(t)-v_{2}(t)\right|\right), \\
& \left|h_{2}\left(t, u_{1}(t), v_{1}(t)\right)-h_{2}\left(t, u_{2}(t), v_{2}(t)\right)\right| \\
& \quad \leq \frac{t}{2000}\left(\left|\arctan u_{1}(t)-\arctan u_{2}(t)\right|+\left|\arcsin v_{1}(t)-\arcsin v_{2}(t)\right|\right) \\
& \quad \leq \frac{t}{2000}\left(\left|u_{1}(t)-u_{2}(t)\right|+\left|v_{1}(t)-v_{2}(t)\right|\right),
\end{aligned}
$$

and

$$
\begin{aligned}
& \left|h_{3}\left(t, u_{1}(t), v_{1}(t)\right)-h_{3}\left(t, u_{2}(t), v_{2}(t)\right)\right| \\
& \quad \leq \frac{t}{100}\left(\left|\sin u_{1}(t)-\sin u_{2}(t)\right|+\left|v_{1}(t)-v_{2}(t)\right|\right) \\
& \quad \leq \frac{t}{100}\left(\left|u_{1}(t)-u_{2}(t)\right|+\left|v_{1}(t)-v_{2}(t)\right|\right) .
\end{aligned}
$$


Hence, $\sigma_{1}(t)=\frac{t}{10}, \sigma_{2}(t)=\frac{t}{2000}$, and $\sigma_{3}(t)=\frac{t}{100}$ where $\left\|\sigma_{1}\right\|=\frac{1}{10},\left\|\sigma_{2}\right\|=\frac{1}{2000}$, and $\left\|\sigma_{3}\right\|=$ $\frac{1}{100}$. Define the continuous and nondecreasing functions $\phi_{i}: \mathbb{R}^{+} \rightarrow \mathbb{R}$ by $\phi_{1}(u)=\phi_{2}(u)=$ $\phi_{3}(u)=u$ for all $u \in \mathbb{R}^{+}$. Then we get

$$
\begin{aligned}
\left|h_{1}\left(t, u(t), u^{\prime}(t)\right)\right| & \leq \frac{t}{10}\left(|u|+\left|\arcsin u^{\prime}\right|\right) \\
& \leq \frac{t}{10}\left(|u|+\left|u^{\prime}\right|\right)=\frac{t}{10} \phi_{1}\left(|u|+\left|u^{\prime}\right|\right), \\
\left|h_{2}\left(t, u(t), u^{\prime}(t)\right)\right| & \leq \frac{t}{2000}\left(|\arctan u|+\left|\arcsin u^{\prime}\right|\right) \\
& \leq \frac{t}{2000}\left(|u|+\left|u^{\prime}\right|\right)=\frac{t}{2000} \phi_{2}\left(|u|+\left|u^{\prime}\right|\right), \\
\left|h_{3}\left(t, u(t), u^{\prime}(t)\right)\right| & \leq \frac{t}{100}\left(|\sin u|+\left|u^{\prime}\right|\right) \\
& \leq \frac{t}{100}\left(|u|+\left|u^{\prime}\right|\right)=\frac{t}{100} \phi_{3}\left(|u|+\left|u^{\prime}\right|\right),
\end{aligned}
$$

where continuous functions $\delta_{i}:[0,1] \rightarrow \mathbb{R}$ are defined by $\delta_{1}(t)=\frac{t}{10}, \delta_{2}(t)=\frac{t}{2000}$, and $\delta_{3}(t)=$ $\frac{t}{100}$. According to the obtained values, we get $\Delta_{1}^{*} \simeq 0.4808, \Delta_{2}^{*} \simeq 0.2486$, and so $\Delta_{1}^{*}+\Delta_{1}^{*} \simeq$ 0.7294. Hence, $k:=\left(\Delta_{1}^{*}+\Delta_{2}^{*}\right) \sum_{i=1}^{3}\left\|\sigma_{i}\right\|=\left(\Delta_{1}^{*}+\Delta_{2}^{*}\right)\left(\left\|\sigma_{1}\right\|+\left\|\sigma_{2}\right\|+\left\|\sigma_{3}\right\|\right) \simeq 0.08059<1$. Now, by using Theorem 5 , we conclude that the fractional boundary value problems (9)(10) have a solution.

\section{Conclusion}

With the development and expansion of some dynamical and industrial systems such as gas transmission lines, water pipelines, the expansive growth of computer networks, structure of molecules in medicine and biology, etc., new descriptive models have emerged for studying the related processes designed by specialists of these fields. Due to the graph representation of these networks, the study of mathematical models described by ordinary or fractional differential equations on special graphs was considered. In this paper, our aim was to extend the idea on more generalized graphs. In this way, we investigated the existence of solutions for some fractional boundary value problems on the ethane graph. We presented two distinct results by considering different conditions on the problems. Also, we provided an example to illustrate our last main result.

\section{Acknowledgements}

The authors were supported by Azarbaijan Shahid Madani University. The authors express their gratitude to dear unknown referees for their helpful suggestions which improved the final version of this paper.

\section{Funding}

Not applicable.

Availability of data and materials

Data sharing not applicable to this article as no datasets were generated or analyzed during the current study.

Ethics approval and consent to participate

Not applicable.

Competing interests

The authors declare that they have no competing interests. 
Authors' contributions

The authors declare that the study was realized in collaboration with equal responsibility. All authors read and approved the final manuscript.

\section{Publisher's Note}

Springer Nature remains neutral with regard to jurisdictional claims in published maps and institutional affiliations.

Received: 19 March 2020 Accepted: 28 May 2020 Published online: 09 June 2020

\section{References}

1. Alizadeh, S., Baleanu, D., Rezapour, S.: Analyzing transient response of the parallel RCL circuit by using the Caputo-Fabrizio fractional derivative. Adv. Differ. Equ. 2020, 55 (2020). https://doi.org/10.1186/s13662-020-2527-0

2. Baleanu, D., Jajarmi, A., Mohammadi, H., Rezapour, S.: Analysis of the human liver model with Caputo-Fabrizio fractional derivative. Chaos Solitons Fractals 134, 109705 (2020)

3. Baleanu, D., Mohammadi, H., Rezapour, S.: Mathematical theoretical study of a particular system of Caputo-Fabrizio fractional differential equations for the rubella disease model. Adv. Differ. Equ. 2020, 184 (2020). https://doi.org/10.1186/s13662-020-02614-z

4. Baleanu, D., Mohammadi, H., Rezapour, S.: Analysis of the model of HIV-1 infection of $c d 4^{+}$T-cell with a new approach of fractional derivative. Adv. Differ. Equ. 2020, 71 (2020)

5. Kumar, D., Singh, J., Tanwar, K., Baleanu, D.: A new fractional exothermic reactions model having constant heat source in porous media with power, exponential and Mittag-Leffler laws. Int. J. Heat Mass Transf. 138, 1222-1227 (2019). https://doi.org/10.1016/j.ijheatmasstransfer.2019.04.094

6. Goswami, A., Singh, J., Kumar, D., Sunshila: An efficient analytical approach for fractional equal width equations describing hydro-magnetic waves in cold plasma. Physica A 524, 563-575 (2019). https://doi.org/10.1016/j.physa.2019.04.058

7. Bhatter, S., Mathur, A., Kumar, D., Nisar, K.S., Singh, J.: Fractional modified Kawahara equation with Mittag-Leffler law. Chaos Solitons Fractals 131, 109508 (2020). https://doi.org/10.1016/j.chaos.2019.109508

8. Kumar, D., Singh, J., Baleanu, D.: On the analysis of vibration equation involving a fractional derivative with Mittag-Leffler law. Math. Methods Appl. Sci. 43(1), 443-457 (2019). https://doi.org/10.1002/mma.5903

9. Singh, J., Kumar, D., Baleanu, D.: A new analysis of fractional fish farm model associated with Mittag-Leffler type kernel. Int. J. Biomath. 13(2), 2050010 (2020). https://doi.org/10.1142/S1793524520500102

10. Alijani, Z., Baleanu, D., Shiri, B., Wu, G.: Spline collocation methods for systems of fuzzy fractional differential equations Chaos Solitons Fractals 131, 109510 (2020)

11. Dadkhah, E., Ghaffarzadeh, H., Shiri, B., Katebi, J.: Spline collocation methods for seismic analysis of multiple degree of freedom systems with visco-elastic dampers using fractional models. J. Vib. Control (2020). https://doi.org/10.1177/1077546319898570

12. Dadkhah, E., Shiri, B., Ghaffarzadeh, H., Baleanu, D.: Visco-elastic dampers in structural buildings and numerical solution with spline collocation methods. J. Appl. Math. Comput. 63, 29-57 (2020)

13. Shiri, B., Baleanu, D.: System of fractional differential algebraic equations with applications. Chaos Solitons Fractals 120, 203-212 (2019)

14. Baleanu, D., Shiri, B., Srivastava, H., Al Qurashi, M.: A Chebyshev spectral method based on operational matrix for fractional differential equations involving non-singular Mittag-Leffler kernel. Adv. Differ. Equ. 2018, 353 (2018)

15. Baleanu, D., Shiri, B.: Collocation methods for fractional differential equations involving non-singular kernel. Chaos Solitons Fractals 116, 136-145 (2018)

16. Baleanu, D., Ghafarnezhad, K., Rezapour, S.: On a three steps crisis integro-differential equation. Adv. Differ. Equ. 2019, $153(2019)$

17. Baleanu, D., Rezapour, S., Mohammadi, H.: Some existence results on nonlinear fractional differential equations Philos. Trans. - Royal Soc., Math. Phys. Eng. Sci. 371, 20120144 (2013). https://doi.org/10.1098/rsta.2012.0144

18. Aydogan, M., Baleanu, D., Mousalou, A., Rezapour, S.: On high order fractional integro-differential equations including the Caputo-Fabrizio derivative. Bound. Value Probl. 2018, 90 (2018). https://doi.org/10.1186/s13661-018-1008-9

19. Aydogan, M., Baleanu, D., Mousalou, A., Rezapour, S.: On approximate solutions for two higher-order Caputo-Fabrizio fractional integro-differential equations. Adv. Differ. Equ. 2017, 221 (2017). https://doi.org/10.1186/s13662-017-1258-3

20. Baleanu, D., Rezapour, S., Saberpour, Z:: On fractional integro-differential inclusions via the extended fractional Caputo-Fabrizio derivation. Bound. Value Probl. 2019, 79 (2019). https://doi.org/10.1186/s13661-019-1194-0

21. Delbosco, D.: Fractional calculus and function spaces. J. Fract. Calc. 6, 45-53 (1994)

22. Zhang, S.: The existence of a positive solution for a nonlinear fractional differential equation. J. Math. Anal. Appl. 252(2), 804-812 (2000). https://doi.org/10.1006/jmaa.2000.7123

23. Bai, Z., Lü, H.: Positive solutions for boundary value problem of nonlinear fractional differential equation. J. Math. Anal. Appl. 311(2), 495-505 (2005). https://doi.org/10.1016/j.jmaa.2005.02.052

24. Bai, Z., Lü, H.: Positive solutions for boundary value problem of nonlinear fractional differential equation. J. Math. Anal. Appl. 311(2), 495-505 (2005). https://doi.org/10.1016/j.jmaa.2005.02.052

25. Kochubei, A.N.: Distributed order calculus and equations of ultraslow diffusion. J. Math. Anal. Appl. 340(1), 252-281 (2008). https://doi.org/10.1016/j.jmaa.2007.08.024

26. Leggett, R.W., Williams, L.R.: Multiple positive fixed points of nonlinear operators on ordered Banach spaces. Indiana Univ. Math. J. 28(4), 673-688 (1979)

27. Agarwal, R.P. O'Regan, D. Staněk, S.: The existence of solutions for a nonlinear mixed problem of singular fractional differential equations. Math. Nachr. 285(1), 27-41 (2012). https://doi.org/10.1002/mana.201000043

28. Agarwal, R.P., O’Regan, D., Staněk, S.: Positive solutions for Dirichlet problems of singular nonlinear fractional differential equations. J. Math. Anal. Appl. 371(1), 57-68 (2010). https://doi.org/10.1016/j.jmaa.2010.04.034 
29. Jiang, M., Zhong, S.: Existence of solutions for nonlinear fractional q-difference equations with Riemann-Liouville type q-derivatives. J. Appl. Math. Comput. 47(1-2), 429-459 (2015). https://doi.org/10.1007/s12190-014-0784-3

30. Zhang, X., Zhong, Q.: Multiple positive solutions for nonlocal boundary value problems of singular fractional differential equations. Bound. Value Probl. 2016, 65 (2016). https://doi.org/10.1186/s13661-016-0572-0

31. Zhou, H., Alzabut, J., Yang, L.: On fractional Langevin differential equations with anti-periodic boundary conditions. Eur. Phys. J. Spec. Top. 226, 3577-3590 (2017). https://doi.org/10.1140/epjst/e2018-00082-0

32. Xu, X., Jiang, D., Yuan, C.: Multiple positive solutions for the boundary value problem of a nonlinear fractional differential equation. Nonlinear Anal. 71, 4676-4688 (2009). https://doi.org/10.1016/..na.2009.03.030

33. Ahmad, B., Nieto, J.J.: Riemann-Liouville fractional integro-differential equations with fractional nonlocal integral boundary conditions. Bound. Value Probl. 2011, 36 (2011). https://doi.org/10.1186/1687-2770-2011-36

34. Su, X., Zhang, S.: Solutions to boundary value problems for nonlinear differential equations of fractional order. Electron. J. Differ. Equ. 2009, 26 (2009)

35. Ragusa, M.A.: Cauchy-Dirichlet problem associated to divergence form parabolic equations. Commun. Contemp. Math. 6(3), 377-393 (2004). https://doi.org/10.1142/S0219199704001392

36. Chidouh, A., Torres, D.: Existence of positive solutions to a discrete fractional boundary value problem and corresponding Lyapunov-type inequalities. Opusc. Math. 38(1), 31-40 (2018). https://doi.org/10.7494/OpMath.2018.38.1.31

37. Denton, Z., Ramírez, J.D.: Existence of minimal and maximal solutions to RL fractional integro-differential initial value problems. Opusc. Math. 37(5), 705-724 (2017). https://doi.org/10.7494/OpMath.2017.37.5.705

38. Liu, Y: A new method for converting boundary value problems for impulsive fractional differential equations to integral equations and its applications. Adv. Nonlinear Anal. 8(1), 386-454 (2019). https://doi.org/10.1515/anona-2016-0064

39. Wang, Y., Liu, L.: Necessary and sufficient condition for the existence of positive solution to singular fractional differential equations. Adv. Differ. Equ. 2015, 207 (2015)

40. Wang, Y.: Positive solutions for a class of two-term fractional differential equations with multipoint boundary value conditions. Adv. Differ. Equ. 2019, 304 (2019). https://doi.org/10.1186/s13662-019-2250-x

41. Wang, Y.: Necessary conditions for the existence of positive solutions to fractional boundary value problems at resonance. Appl. Math. Lett. 97, 34-40 (2019). https://doi.org/10.1016/j.aml.2019.05.007

42. Bungardi, S., Cardinali, T., Rubbioni, P.: Nonlocal semi-linear integro-differential inclusions via vectorial measures of non-compactness. Appl. Anal. 96(15), 2526-2544 (2015)

43. Ndaírou, F., Area, I., Nieto, J.J., Torres, D.F.M.: Mathematical modeling of COVID-19 transmission dynamics with a case study of Wuhan. Chaos Solitons Fractals 135, 109846 (2020)

44. Kucche, K.D., Nieto, J.J., Venktesh, V.: Theory of nonlinear implicit fractional differential equations. Differ. Equ. Dyn. Syst. 28(1), 1-17 (2020)

45. Ahmad, B., Alruwaily, Y., Alsaedi, A., Nieto, J.J.: Fractional integro-differential equations with dual anti-periodic boundary conditions. Differ. Integral Equ. 33(3-4), 181-206 (2020)

46. Nisar, K.S., Suthar, D.L., Agarwal, R., Purohit, S.D.: Fractional calculus operators with Appell function kernels applied to Srivastava polynomials and extended Mittag-Leffler function. Adv. Differ. Equ. 2020, 148 (2020)

47. Agarwal, R., Golev, A., Hristova, S., O'Regan, D., Stefanova, K.: Iterative techniques with computer realization for the initial value problem for Caputo fractional differential equations. J. Appl. Math. Comput. 58(1-2), 433-467 (2018)

48. Hristova, S., Agarwal, R., O'Regan, D.: Explicit solutions of initial value problems for systems of linear Riemann-Liouville fractional differential equations with constant delay. Adv. Differ. Equ. 2020, 180 (2020)

49. Wang, X., Li, X., Huang, N., O'Regan, D.: Asymptotical consensus of fractional-order multi-agent systems with current and delay states. Appl. Math. Mech. 40(11), 1677-1694 (2019)

50. Song, J., Xia, Y., Bai, Y., Cai, Y., O'Regan, D.: A non-autonomous Leslie-Gower model with Holling type IV functional response and harvesting complexity. Adv. Differ. Equ. 2019, 299 (2019)

51. Agarwal, P., Chand, M., Baleanu, D., O'Regan, D., Jain, S.: On the solutions of certain fractional kinetic equations involving k-Mittag-Leffler function. Adv. Differ. Equ. 2018, 249 (2018)

52. Lumer, G.: Connecting of local operators and evolution equations on a network. Lect. Notes Math. 787, 219-234 (1985)

53. Nicaise, S.: Some results on spectral theory over networks applied to nerve impulses transmission. Lect. Notes Math $1171,532-541$ (1985)

54. Zavgorodnii, M.G., Pokornyi, Y.V.: On the spectrum of second-order boundary value problems on spatial networks. Usp. Mat. Nauk 44, 220-221 (1989)

55. Gordeziani, D.G., Kupreishvli, M., Meladze, H.V., Davitashvili, T.D.: On the solution of boundary value problem for differential equations given in graphs. Appl. Math. Lett. 13, 80-91 (2008)

56. Graef, J.R., Kong, L.J., Wang, M.: Existence and uniqueness of solutions for a fractional boundary value problem on a graph. Fract. Calc. Appl. Anal. 17, 499-510 (2014)

57. Mehandiratta, V., Mehra, M., Leugering, G.: Existence and uniqueness results for a nonlinear Caputo fractional boundary value problem on a star graph. J. Math. Anal. Appl. 477, 1243-1264 (2019)

58. Podlubny, l.: Fractional Differential Equations. Academic Press, New York (1999)

59. Samko, G., Kilbas, A., Marichev, O.: Fractional Integrals and Derivatives: Theory and Applications. Gordon \& Breach, New York (1993)

60. Miller, K.S., Ross, B.: An Introduction to Fractional Calculus and Fractional Differential Equations. Wiley, New York (1993)

61. Smart, D.R.: Fixed Point Theorems. Cambridge University Press, Cambridge (1990) 